\title{
The maximal unipotent finite quotient, unusual torsion in Fano threefolds, and exceptional Enriques surfaces
}

\author{
Andrea Fanelli and Stefan Schröer
}

\begin{abstract}
We introduce and study the maximal unipotent finite quotient for algebraic group schemes in positive characteristics. Applied to Picard schemes, this quotient encodes unusual torsion. We construct integral Fano threefolds where such unusual torsion actually appears. The existence of such threefolds is surprising, because the unusual torsion vanishes for del Pezzo surfaces. Our construction relies on the theory of exceptional Enriques surfaces, as developed by Ekedahl and Shepherd-Barron.
\end{abstract}

Keywords. Fano varieties, Enriques surfaces, group schemes

2020 Mathematics Subject Classification. 14J45, 14J28, 14L15, 14C22

[Français]

Le quotient fini unipotent maximal, torsion inhabituelle dans les solides de Fano et surfaces d'Enriques exceptionnelles.

Résumé. Nous introduisons et étudions le quotient fini unipotent maximal pour les schémas en groupes algébriques en caractéristique positive. Appliqué aux schémas de Picard, ce quotient encode la torsion inhabituelle. Nous construisons des solides de Fano intègres qui font effectivement apparaître cette torsion inhabituelle. L'existence de ces solides est surprenante, compte tenu du fait que la torsion inhabituelle s'annule pour les surfaces de Del Pezzo. Notre construction repose sur la théorie des surfaces d'Enriques développée par Ekedahl et Shepherd-Barron.

Received by the Editors on February 24, 2020, and in final form on May 25, 2020.

Accepted on July 3, 2020.

Andrea Fanelli

Institut de mathématiques de Bordeaux (IMB), CNRS, Université de Bordeaux, 33405 Talence cedex, France

e-mail: andrea.fanelli.1@u-bordeaux.fr

Stefan Schröer

Mathematisches Institut, Heinrich-Heine-Universität, 40204 Düsseldorf, Germany

e-mail: schroeer@math.uni-duesseldorf.de

This research was conducted in the framework of the research training group GRK 2240: Algebro-geometric Methods in Algebra, Arithmetic and Topology; we wish to thank the Deutsche Forschungsgemeinschaft for financial support. The first-named author is currently funded by the Fondation Mathématique Jacques Hadamard.

(C) by the author(s)

This work is licensed under http://creativecommons.org/licenses/by-sa/4.0/ 


\section{Contents}

1. The maximal finite unipotent quotient . . . . . . . . . . . . . . . . . . . . . 4

2. Picard scheme and Bockstein operators . . . . . . . . . . . . . . . . . . . . . . . . . . 9

3. The case of surfaces. . . . . . . . . . . . . . . . . . . . . . . . . . . . . 11

4. Enriques surfaces and del Pezzo surfaces . . . . . . . . . . . . . . . . . . . . 13

5. Exceptional Enriques surfaces. . . . . . . . . . . . . . . . . . . . . . . . . 16

6. Cones and Fano varieties . . . . . . . . . . . . . . . . . . . . . . . . . . 21

7. Fano threefolds with unusual torsion . . . . . . . . . . . . . . . . . . . . . . 23

References. . . . . . . . . . . . . . . . . . . . . . . . . . 26

\section{Introduction}

In algebraic geometry over ground fields $k$ of characteristic $p>0$, unusual behavior of certain algebraic schemes is often reflected by the structure of unipotent torsion originating from the Picard group. For example, an elliptic curve $E$ is supersingular if and only if the kernel $E[p]$ for multiplication-by- $p$ is unipotent. An even more instructive case are Enriques surfaces $Y$, which have $c_{1}=0$ and $b_{2}=10$. Then the Picard scheme $P=\operatorname{Pic}_{Y / k}^{\tau}$ of numerically trivial invertible sheaves has order two. In characteristic $p=2$, this gives the three possibilities. In case $P=\mu_{2}$, the Enriques surface is called ordinary, and behaves like in characteristic zero. Otherwise, we have $P=\mathbb{Z} / 2 \mathbb{Z}$ or $P=\alpha_{2}$, which is a unipotent group scheme, and $Y$ is a simply-connected Enriques surfaces. Their geometry and deformation theory is more difficult to understand. The crucial difference to the case of elliptic curves is that the unipotent torsion can be regarded as a quotient object, and not only as a subobject, which makes it more "unusual".

The first goal of this paper is to introduce a general measure for unipotent torsion, the maximal finite unipotent quotient $\Upsilon_{Y / k}=\Upsilon_{P}$ of the algebraic group scheme $P=\operatorname{Pic}_{Y / k}^{\tau}$. Over algebraically closed fields, this comprises the $p$-primary torsion part of the Néron-Severi group NS $(Y)$ and the part of the local group scheme $P^{0} / P_{\text {red }}^{0}$ whose Cartier dual is also local. Such finite group schemes are often called local-local. This actually extends to algebraic group scheme that are not necessarily commutative. Our approach builds on the work of Brion [Bril7]. It turns out that $\Upsilon_{Y / k}$ is useful in various situations. For example, it easily explains that the reduced part of an algebraic group scheme is not necessarily a subgroup scheme. Of course, this may only happen over imperfect fields.

The second goal is to construct Fano varieties whose Picard scheme actually contains such unipotent torsion. Roughly speaking, a Fano variety is a Gorenstein scheme $Y$ that is proper and equi-dimensional, and whose dualizing sheaf $\omega_{Y}$ is anti-ample. This notion indeed goes back to Fano [Fan31]. Usually, one also assumes that $Y$ is integral. We write $n=\operatorname{dim}(Y)$ for the dimension. The structure and classification of Fano varieties is an interesting subject of its own. Fano varieties play an important role in representation theory, because proper homogeneous spaces $Y=G / H$ for linear groups schemes in characteristic $p=0$ are Fano varieties. Moreover, they are crucial for the minimal model program, because they arise as generic fibers $Y=X_{\eta}$ in Mori fibrations $X \rightarrow B$. 
Suppose that $Y$ is a smooth Fano variety in characteristic zero. Then

$$
H^{i}\left(Y, \mathscr{O}_{Y}\right)=H^{i}\left(Y, \Omega_{Y / k}^{n} \otimes \omega_{Y}^{\otimes-1}\right)=0
$$

for $i>0$, by Kodaira-Akizuki-Nakano Vanishing (see [DI87] for an algebraic proof). In particular, the Lie algebra $H^{1}\left(Y, \mathscr{O}_{Y}\right)$ of the Picard scheme $\mathrm{Pic}_{Y / k}$ vanishes, so that the connected component $\mathrm{Pic}_{Y / k}^{0}$ is trivial. Moreover, the Euler characteristic is $\chi\left(\mathscr{O}_{Y}\right)=1$, whence all connected étale coverings are trivial. Consequently, the algebraic fundamental group vanishes, and the Néron-Severi group NS $(Y)$ is torsion-free. Summing up, the group scheme $\operatorname{Pic}_{Y / k}^{\tau}$ of numerically trivial invertible sheaves vanishes. Its fundamental role was emphasized by Grothendieck [FGA4].

It is a natural question to what extent the vanishing for $\operatorname{Pic}_{Y / k}^{\tau}$ holds true in positive characteristics, or for singular Fano varieties. Vanishing holds in dimension $n=1$ for integral Gorenstein curves with $\omega_{Y}^{\otimes-1}$ ample, basically by Riemann-Roch. In dimension $n=2$, the classification of regular del Pezzo surfaces is independent of the characteristic, and we again have vanishing. For normal del Pezzo surfaces, the second author observed in [Sch01] that $h^{1}\left(\mathscr{O}_{Y}\right)=0$. The situation becomes much more challenging for non-normal del Pezzo surfaces. Here Reid [Rei94] constructed for each prime $p>0$ examples with $h^{1}\left(\mathscr{O}_{Y}\right) \neq 0$. In [Sch07], some normal locally factorial del Pezzo surfaces over imperfect fields in characteristic $p=2$ with $h^{1}\left(\mathscr{O}_{Y}\right) \neq 0$ were constructed, and even regular examples exist, as shown by Maddock [Mad16]. For smooth Fano threefolds, the vanishing of $\operatorname{Pic}_{Y / k}^{\tau}$ was shown by Shepherd-Barron [SB97]. However, Cascini and Tanaka [CT19] constructed a klt Fano threefold in characteristic $p=2$ with $h^{2}\left(\mathscr{O}_{Y}\right) \neq 0$; other examples with $p \geq 3$ were found by Bernasconi [Ber19].

Moreover, Tanaka [Tan16] constructed Mori fibrations $X \rightarrow B$ on threefolds in characteristic $p=2,3$ where the generic fiber $Y=X_{\eta}$ is a normal del Pezzo surface whose Picard group contains elements of order $p=2,3$. Very recently, Bernasconi and Tanaka [BT20] provided effective bounds for the torsion on del Pezzo-type surfaces over imperfect fields.

However, in all the above examples the maximal unipotent finite quotient $\Upsilon_{Y / k}$ of Pic P $_{Y / k}^{\tau}$ still vanishes. We therefore regard $\Upsilon_{Y / k}$ as a measure for unusual torsion in Fano varieties. The main results of this paper are as follows. First, we give general criteria for the vanishing of $\Upsilon_{Y / k}$, which relies on the theory of Bockstein operators:

Theorem. (see Thm. 2.2) We have $\Upsilon_{Y / k}^{0}=0$ provided the Frobenius map on $H^{2}\left(Y, \mathscr{O}_{Y}\right)$ has maximal Hasse-Witt rank.

From this we deduce in Theorem 3.3 that $\Upsilon_{Y / k}=0$ for any reduced surface whose dualizing sheaf $\omega_{Y}$ is negative, in a suitable sense. Note that this requires no further restrictions on the singularities. It applies in particular to del Pezzo surfaces. In light of this, it is surprising that unusual torsion does appear in dimension $n \geq 3$ :

Theorem. (see Thm. 7.3) There are integral Fano threefolds $Y$ in characteristic two such that $\Upsilon_{Y / k}=\operatorname{Pic}_{Y / k}^{\tau}$ is isomorphic to the group scheme $\mathbb{Z} / 2 \mathbb{Z}$ or $\alpha_{2}$.

The construction relies on the theory of exceptional Enriques surfaces, as developed by Ekedahl and Shepherd-Barron [ESB04]. These are simply-connected Enriques surfaces containing very strange configurations of $(-2)$-curves. The geometry of such surfaces was already studied in the monograph of Cossec and Dolgachev ([CD89], Chapter III, §3-4), although their existence was established only later by Salomonsson [Sal03].

The idea for our construction is rather simple: start with an exceptional Enriques surface $S$ that contains exactly ten (-2)-curves with a certain dual graph. We then contract all but one of these curves, creating a normal Enriques surface $Z$. Its normalized K3-like covering $Z^{\prime} \rightarrow Z$ turns out to be a normal del Pezzo surface with a unique singularity, which is a rational double point of type $D_{5}$. This relies on the classification of normal del Pezzo surfaces, as explained by Dolgachev [Dol12]. We take the $\mathbb{P}^{1}$-bundle $X=\mathbb{P}(\mathscr{E})$ for 
the locally free sheaf $\mathscr{E}=\mathscr{O}_{Z^{\prime}} \oplus \omega_{Z^{\prime}}^{\otimes-1}$, contract the negative section $E=\mathbb{P}\left(\omega_{Z^{\prime}}^{\otimes-1}\right)$, and denormalize along the positive section $Z^{\prime}=\mathbb{P}\left(\mathscr{O}_{Z}\right)$ with respect to the purely inseparable double covering $v: Z^{\prime} \rightarrow Z$. The resulting scheme $Y=X \amalg_{Z} Z^{\prime}$ is an integral Fano threefold with Euler characteristic, degree and index

$$
\chi\left(\mathscr{O}_{Y}\right)=1, \quad \operatorname{deg}(Y)=4 \quad \text { and } \operatorname{ind}(Y)=1 .
$$

A detailed analysis of the normalized K3-like covering $v: Z^{\prime} \rightarrow Z$ reveals that the morphism is flat, except over a rational double point $a \in Z$ of type $E_{8}$. From this we infer that our integral Fano threefold $Y$ has invertible dualizing sheaf and satisfies Serre Condition $\left(S_{2}\right)$, but fails to be Cohen-Macaulay. Such schemes might be called quasi-Fano varieties, and deserve further study.

Note that recently Totaro [Tot19] constructed three-dimensional terminal singularities in positive characteristics that are not Cohen-Macaulay, and further examples were constructed by Yasuda [Yas19]. In turn, the above examples of non-normal Fano threefolds $Y$ may admit twisted forms $Y^{\prime}$ whose local rings are normal $\mathbb{Q}$-factorial klt singularities, and thus could occur as generic fibers in Mori fiber spaces. See [FS20] for our analysis of non-normal del Pezzo surfaces having twisted forms whose local rings are regular.

The paper is organized as follows: In Section 1 we discuss various smallest normal subgroup schemes $N \subset G$ whose quotients have certain properties, introduce the maximal finite unipotent quotient $\Upsilon_{G}=G / N$, and establish its basic properties. We apply this in Section 2 to Picard schemes $G=\operatorname{Pic}_{Y / k}^{\tau}$, and give general criteria for the vanishing of $\Upsilon_{Y / k}=\Upsilon_{G}$. In Section 3 we show that $\Upsilon_{Y / k}$ vanishes for reduced surfaces with negative dualizing sheaf. In Section 4 we recall various notions of Enriques surfaces, and describe how del Pezzo surfaces arise as normalization of K3-like coverings. The following Section 5 contains a detailed analysis for the case of exceptional Enriques surfaces of type $T_{2,3,7}$. In Section 6 we discuss the cone construction and its Gorenstein properties, and show how it leads to new Fano varieties. The final Section 7 contains the construction of integral Fano threefolds whose maximal finite unipotent quotient $\Upsilon_{Y / k}$ is non-trivial.

Acknowledgements: We like to thank Fabio Bernasconi, Michel Brion, Igor Dolgachev, Hiromu Tanaka and the referee for many valuable suggestions.

\section{The maximal finite unipotent quotient}

In order to study unusual torsion in Picard groups, we shall introduce the maximal finite unipotent quotient for general algebraic group schemes, which are not necessarily commutative. These results seem to be of independent interest, and mainly rely on the theory of algebraic groups.

Let $k$ be a ground field of characteristic $p \geq 0$. An algebraic group scheme is a group scheme $G$ where the structure morphism $G \rightarrow \operatorname{Spec}(k)$ is of finite type. Note that the underlying scheme is automatically separated. We say that $G$ is finite if the structure morphism is finite. Then the order is defined as $\operatorname{ord}(G)=h^{0}\left(\mathscr{O}_{G}\right)=\operatorname{dim}_{k} H^{0}\left(G, \mathscr{O}_{G}\right)$. One says that $G$ is of multiplicative type if the base-change $G \otimes k^{\text {alg }}$ to the algebraic closure is isomorphic to Spec $k^{\text {alg }}[M]$, where $M$ is a finitely generated abelian group. In other words, $G$ is commutative and the base-change has a filtration whose subquotients are isomorphic to the multiplicative group $\mathbb{G}_{m}$, or the constant groups $\mu_{l}=\mathbb{Z} / l \mathbb{Z}$ for some prime $l \neq p$, or the local group scheme $\mu_{p}$. One also says that $G$ is multiplicative. The algebraic group $G$ is called unipotent if $G \otimes k^{\text {alg }}$ admits a filtration whose subquotients are isomorphic to subgroup schemes of the additive group $\mathbb{G}_{a}$. In characteristic $p>0$, this means that after refinement the subquotients are $\mathbb{G}_{a}$, the constant group $\mathbb{Z} / p \mathbb{Z}$ or the local group scheme $\alpha_{p}$. Note that unipotent group schemes are not necessarily commutative. For more on these notions, see [SGA3-II, exposé IX and exposé XVII].

Theorem 1.1. For each of the following properties (i)-(vi) and each algebraic group scheme $G$, there is a smallest normal subgroup scheme $N \subset G$ such that the quotient $G / N$ has the property in question: 
(i) étale;

(ii) affine;

(iii) proper; (iv) finite;

(v) finite and multiplicative;

(vi) finite and unipotent.

Moreover, the subgroup schemes $N \subset G$ and the quotients $G / N$ commute with separable field extensions $k \subset k^{\prime}$.

Proof. By [SGA3-I, Exposé $\mathrm{IV}_{\mathrm{A}}$, Section 2], the connected component $G^{0} \subset G$ of the origin is a normal subgroup scheme such that $G / G^{0}$ is étale, and it is indeed the smallest. According to Brion's analysis ([Bril7, Theorem 1 and Theorem 2]), there are the smallest normal subgroup schemes $N_{1}, N_{2} \subset G$ such that the resulting quotients are affine and proper, respectively. Let $N \subset G$ be the normal subgroup scheme generated by $N_{1}$ and $N_{2}$. Then the resulting quotient $G / N$ is both proper and affine, hence finite. Moreover, every homomorphism $G \rightarrow K$ into some finite group scheme contains $N_{1}$ and $N_{2}$ in its kernel. It follows that $N$ is the desired smallest normal subgroup scheme with finite quotient. This settles the cases (i)-(iv).

Consider the ordered family of normal subgroup schemes $H_{\lambda} \subset G, \lambda \in L$ whose quotients $G / H_{\lambda}$ are finite and unipotent. The group scheme $G$ itself belongs to this family, and each member contains $N$. We first check that for any two members $H_{\lambda}$ and $H_{\mu}$, the intersection $K=H_{\lambda} \cap H_{\mu}$ also belongs to the family. We have an exact sequence

$$
0 \longrightarrow H_{\lambda} / K \longrightarrow G / K \longrightarrow G / H_{\lambda} \longrightarrow 0
$$

By the Isomorphism Theorem, the term on the left is isomorphic to $\left(H_{\lambda} \cdot H_{\mu}\right) / H_{\mu}$, which is contained in $G / H_{\mu}$. The latter is finite and unipotent, so the same holds for the subgroup scheme $\left(H_{\lambda} \cdot H_{\mu}\right) / H_{\mu}$ and the extension $G / K$.

Seeking a contradiction, we assume that there is no smallest member. Since the family is filtered, this means that it contains an infinite descending sequence $H_{0} \supsetneqq H_{1} \supsetneqq \ldots$ such that the quotients $U_{n}=G / H_{n}$ have unbounded orders. On the other hand, all of them are quotients of the finite group scheme $G / N$, hence the orders are bounded, contradiction. Hence, there is a smallest normal subgroup scheme whose quotient is finite and unipotent. This settles (vi). The argument for $(\mathrm{v})$ is similar and left to the reader.

We now prove the second part of the assertion: let $k \subset k^{\prime}$ be a separable extension, and $N^{\prime} \subset G^{\prime} \otimes k^{\prime}$ be the smallest subgroup scheme over $k^{\prime}$ such that the quotient has the property $\mathcal{P}$ in question. This gives an inclusion $N^{\prime} \subset N \otimes k^{\prime}$, and we have to verify that it is an equality. Suppose first that $k \subset k^{\prime}$ is algebraic. By fpqc descent, it suffices to check $N^{\prime}=N \otimes k^{\prime}$ after enlarging the field extension. It thus suffices to treat the case that $k \subset k^{\prime}$ is Galois, with Galois group $\Gamma=\operatorname{Gal}\left(k^{\prime} / k\right)$. Then for each element $\sigma \in \Gamma$ we have $\sigma\left(N^{\prime}\right)=N^{\prime}$, by the uniqueness of $N^{\prime} \subset G \otimes k^{\prime}$. Galois descent gives a closed subscheme $N_{0} \subset N$ with $N_{0} \otimes k^{\prime}=N^{\prime}$. This subscheme is a subgroup scheme and normal in $G$, and the base-change $G / N_{0} \otimes k^{\prime}=\left(G \otimes k^{\prime}\right) / N^{\prime}$ has property $\mathcal{P}$. For each of the cases (i)-(vi), this implies that $G / N_{0}$ has property $\mathcal{P}$. This gives $N_{0}=N$, and in turn the desired equality $N^{\prime}=N \otimes k^{\prime}$.

Now write $k^{\prime}=\bigcup k_{\lambda}$ as the filtered union of finitely generated subextensions. The structure morphism $G^{\prime} \rightarrow \operatorname{Spec}\left(k^{\prime}\right)$ is of finite presentation. Hence, [EGA4-III, Theorem 8.8.2] ensures that there is some index $\lambda$ so that the closed subscheme $N^{\prime}$ is the base-change of some closed subscheme $N_{\lambda} \subset G \otimes k_{\lambda}$. Moreover, this subscheme is a normal subgroup scheme, and we have $N_{\lambda} \subset N \otimes k_{\lambda}$. This reduces our problem to the case that $k^{\prime}$ is finitely generated. Choose an integral affine scheme $S$ of finite type with function field $\kappa(\eta)=k^{\prime}$. Since $k \subset k^{\prime}$ is separable, the scheme $S$ is geometrically reduced. Passing to some dense open set, we may assume that $S$ is smooth.

Consider the relative group scheme $G_{S}$, with generic fiber $G_{\eta}=G \otimes k^{\prime}$. Seeking a contradiction, we assume that $N^{\prime} \subset N \otimes k^{\prime}$ is not an equality. Then there is a homomorphism $f_{\eta}: G_{\eta} \rightarrow H_{\eta}$ to some algebraic group scheme $H_{\eta}$ having property $\mathcal{P}$, such that $N_{\eta}$ is not contained in the kernel. Again by [EGA4-III, théorème 8.8.2], there is a dense open set $U \subset S$, a relative group scheme $H_{U}$ where the structure morphism $H_{U} \rightarrow U$ is of finite type, and a homomorphism $f_{U}: G_{U} \rightarrow H_{U}$ inducing $f_{\eta}$. If the scheme $H_{\eta}$ is affine, proper or finite, we may assume that the respective property holds for the morphism $H_{U} \rightarrow U$, by [EGA4-III, 
théorème 8.10.5]. Moreover, if $H_{\eta}$ is finite and unipotent, we may assume that there is a finite étale covering $U^{\prime} \rightarrow U$ so that $H \times_{U} U^{\prime}$ is a successive extension of $\mathbb{Z} / p \mathbb{Z}$ and $\alpha_{p}$, such that all fibers $H_{U} \rightarrow U$ are finite and unipotent. The situation for properties (i) and (v) is similar. Summing up, we may assume that all fibers of $H_{U} \rightarrow U$ have the property $\mathcal{P}$ in question. Passing to a dense open set again, we reduce to $U=S$. Consider the $S$-scheme

$$
K=\operatorname{Ker}(f \mid N)=N \times_{G}\left\{e_{S}\right\} .
$$

This is a relative group scheme, and the structure morphism $g: K \rightarrow S$ is of finite type. The generic fiber is equi-dimensional, say of dimension $n=\operatorname{dim}\left(K_{\eta}\right)$. By [SGA3-I, exposé $\mathrm{VI}_{\mathrm{B}}$, proposition 4.1], the set of points $a \in S$ with $\operatorname{dim}\left(K_{a}\right)=n$ is constructible. Replacing $S$ by some dense open set, we may assume that all fibers $K_{a}$ are $n$-dimensional. By Bertini's Theorem ([Jou83, théorème 6.3]), there are closed points $a \in S$ such that the finite extension $k \subset \kappa(a)$ is separable. Since the fiber $H_{a}$ has property $\mathcal{P}$ in question, the kernel $K_{a}$ is trivial, and thus $n=0$. In turn, the morphism $g: K \rightarrow S$ is quasi-finite. By Zariski's Main Theorem, there is a closed embedding $K \subset X$ into some finite $S$-scheme $X$, with $K_{\eta}=X_{\eta}$. After replacing $S$ by a dense open set, we may assume that $g: K \rightarrow S$ is finite, and furthermore flat, say of degree $d=\operatorname{deg}(K / S)$. Looking again at fibers $K_{a}$, we see that $d=1$. In turn, $K_{\eta}$ is trivial, contradiction.

Let us say that $G / N$ is the maximal quotient with the property $\mathcal{P}$ in question. By construction, any homomorphism $G \rightarrow H$ into some algebraic group scheme with property $\mathcal{P}$ uniquely factors over $G / N$. Thus $G / N$ is functorial in $G$, and the functor $G \mapsto G / N$ is the left adjoint for the inclusion $H \mapsto H$ of the category of algebraic group schemes with property $\mathcal{P}$ into the category of all algebraic group schemes.

Given a field extension $k \subset k^{\prime}$, we set $G^{\prime}=G \otimes k^{\prime}$ and let $N^{\prime} \subset G^{\prime}$ be the normal subgroup scheme giving the maximal quotient over $k^{\prime}$. This gives an inclusion $N^{\prime} \subset N \otimes k^{\prime}$, and a resulting base-change map $G^{\prime} / N^{\prime} \rightarrow(G / N) \otimes k^{\prime}$. It may or may not be an isomorphism, as we shall see below. However, we have the following immediate fact:

Proposition 1.2. In the above situation, the base-change map is an epimorphism. In particular, the algebraic group scheme $G / N$ vanishes if $G^{\prime} / N^{\prime}$ vanishes.

The maximal affine quotient is indeed the affine hull in the sense of scheme theory, and written as $G^{\text {aff }}=\operatorname{Spec} \Gamma\left(G, \mathscr{O}_{G}\right)$. The kernel $N$ for the homomorphism $G \rightarrow G^{\text {aff }}$ is anti-affine, which means that the inclusion $k \subset H^{0}\left(N, \mathscr{O}_{N}\right)$ is an equality ([DG70, Chapter III, §3, Theorem 8.2]). This notion was introduced by Brion [Bri09], and implies that $N$ is semi-abelian, and in particular smooth and connected ([Bril7, Proposition 5.5.1]). The maximal étale quotient is usually denoted by $\Phi_{G}=G / G^{0}$, and called the group scheme of components. Both $G^{\text {aff }}$ and $\Phi_{G}$ actually commute with arbitrary field extensions $k \subset k^{\prime}$.

Throughout this paper we are mainly interested in the maximal finite unipotent quotient

$$
\Upsilon_{G}=G / N
$$

Any subgroup scheme of $G$ that is multiplicative, or smooth and connected, or merely integral vanishes in $\Upsilon_{G}$. In fact, the following holds:

Lemma 1.3. Suppose the algebraic group scheme $G$ has a filtration whose subquotients are generated by integral group schemes and group schemes of multiplicative type. Then all homomorphisms $f: G \rightarrow U$ into finite unipotent group schemes $U$ are trivial.

Proof. By induction on the length of the filtration, it suffices to treat the two cases that $G$ is integral, or of multiplicative type. In the latter case, the statement follows from [SGA3-II, exposé XVII, proposition 2.4]. Now suppose that $G$ is integral. Replacing $U$ by the image $\operatorname{Im}(f)=G / \operatorname{Ker}(f)$, we may assume that $f: G \rightarrow U$ is surjective and schematically dominant. Setting $A=H^{0}\left(G, \mathscr{O}_{G}\right)$ and $R=H^{0}\left(U, \mathscr{O}_{U}\right)$, we see that the canonical map $R \rightarrow A$ is injective. These rings are integral, because the scheme $G$ is integral. Moreover, $R$ is an Artin ring, because the group scheme $U$ is finite. The neutral element $e \in U$ shows that the residue field is $R / \mathfrak{m}_{R}=k$. In turn, we have $R=k$, thus $U=\operatorname{Spec}(k)$ is trivial. 
Each algebraic group scheme $G$ yields a Galois representation $G\left(k^{\mathrm{sep}}\right)$ of the Galois group $\Gamma=\mathrm{Gal}\left(k^{\mathrm{sep}} / k\right)$ on the abstract group $G\left(k^{\mathrm{sep}}\right)$, which might be infinitely generated. However, this construction yields an equivalence between the category of algebraic group schemes that are étale and continuous Galois representations on finite groups ([SGA4-II, exposé VIII, proposition 2.1]). Note also that for finite group schemes $H$, the group scheme $H^{0}$ is local, and the finite universal homeomorphism $H \rightarrow \Phi_{H}$ yields an equality $H\left(k^{\prime}\right)=\Phi_{H}\left(k^{\prime}\right)$ for all field extensions $k \subset k^{\prime}$. This applies in particular to the maximal finite unipotent quotient $H=\Upsilon_{G}$. The following observation thus computes its group scheme of components:

Proposition 1.4. The Galois module $\Upsilon_{G}\left(k^{\mathrm{sep}}\right)$ is the quotient of the finite group $\Phi_{G}\left(k^{\mathrm{sep}}\right)$ by the subgroup generated by all $l$-Sylow groups for the primes $l \neq p$.

Proof. Base-changing to $k^{\text {sep }}$, we are reduced to the case that the ground field $k$ is separably closed. Write $\Phi_{G}^{\prime}$ for the quotient of the constant group scheme $\Phi_{G}$ by the subgroup generated by the Sylow- $l$-subgroups, and set $U=\Upsilon_{G}$. Then $\Phi_{U}(k)=U(k)$ is a finite $p$-group. It follows that the canonical surjection $\Phi_{G} \rightarrow \Phi_{U}$ factors over $\Phi_{G}^{\prime}$. The resulting map $f: \Phi_{G}^{\prime} \rightarrow \Phi_{U}$ admits a section, by the universal properties of $\Upsilon_{G}$ and $\Phi_{U}$. This section $s: \Phi_{U} \rightarrow \Phi_{G}^{\prime}$ is surjective, because $G \rightarrow \Phi_{G}^{\prime}$ is surjective. In turn, $f$ and $s$ are inverse to each other, and give the desired identification $\Phi_{G}^{\prime}(k)=\Phi_{U}(k)=\Upsilon_{G}(k)$.

It is much more difficult to understand the component of the origin $\Upsilon_{G}^{0}$, because the base-change map $\Upsilon_{G \otimes k^{\prime}} \rightarrow \Upsilon_{G} \otimes k^{\prime}$ might fail to be an isomorphism, for inseparable extensions $k^{\prime}$. Here is a typical example, over imperfect fields $k$ :

Proposition 1.5. Suppose $G$ is an integral group scheme such that some base-change $G^{\prime}=G \otimes k^{\prime}$ becomes isomorphic to $\mathbb{G}_{a} \oplus \alpha_{p}$. Then we have $\Upsilon_{G}=0$, whereas $\Upsilon_{G^{\prime}}=\alpha_{p}$.

Proof. According to Proposition 1.3, the quotient $\Upsilon_{G}$ is trivial, whereas $G^{\prime} \rightarrow \Upsilon_{G^{\prime}}$ is the projection onto the second factor.

For example, $G$ could be the kernel for the homomorphism $h: \mathbb{G}_{a} \times \mathbb{G}_{a} \rightarrow \mathbb{G}_{a}$ given by the additive map $(x, y) \longmapsto x^{p}+t y^{p}$, for some scalar $t \in k$ that is not a $p$-th power. Note that certain fiber products $G=\mathbb{G}_{a} \times_{\mathbb{G}_{a}} \mathbb{G}_{a}$ were systematically studied by Russel [Rus70] to describe twisted forms of the additive group. In our concrete example the compactification in $\mathbb{P}^{2}$ is the Fermat curve with homogeneous equation $X^{p}+t Y^{p}+Z^{p}=0$, as analyzed in [Sch10, §4]. The scheme $G$ is integral, with singular locus $G(k)=\{0\}$, and its normalization is the affine line over the height-one extension $E=k\left(t^{1 / p}\right)$. It has another peculiar feature:

Proposition 1.6. Notation as above. For the connected scheme $H=G \times G$, the closed subscheme $H_{\text {red }} \subset H$ is not a subgroup scheme.

Proof. This already appeared in [SGA3-I, exposé VI, exemple 1.3.2]. Let us give an independent short proof based on our maximal finite unipotent quotient: Suppose $H_{\text {red }}$ were a subgroup scheme. Then $H / H_{\text {red }}$ is a local unipotent group scheme, so the projection factors over $\Upsilon_{H}=\Upsilon_{G} \times \Upsilon_{G}=0$, consequently $H_{\text {red }}=H$. On the other hand, the normalization $\mathbb{A}_{E}^{1} \rightarrow G$ shows that $H$ is birational to the affine plane over the non-reduced ring $E \otimes E=E[t] /\left(t^{p}\right)$, hence $H_{\text {red }} \subsetneq H$, contradiction.

If the reduced part $G_{\text {red }} \subset G$ of an algebraic group scheme is geometrically reduced, then the product $G_{\text {red }} \times G_{\text {red }}$ remains reduced, and the group multiplication factors over $G_{\text {red }}$. In turn, the closed subscheme $G_{\text {red }} \subset G$ is a subgroup scheme. This frequently fails, as we saw above. Other examples for this behavior are the non-split extensions $0 \rightarrow \alpha_{p} \rightarrow G \rightarrow \mathbb{Z} / p \mathbb{Z} \rightarrow 0$, where the fiber $\operatorname{pr}^{-1}(a)$ for the projection pr: $G \rightarrow \mathbb{Z} / p \mathbb{Z}$ is reduced if and only if $a \neq 0$. Such extensions exist over imperfect fields: the abelian group of all central extensions contains the flat cohomology group $H^{1}\left(\operatorname{Spec}(k), \alpha_{p}\right)=k / k^{p}$ as a subgroup, by [DG70, Chapter III, $\S 6$, Proposition in 3.5]. Also note that if $G_{\text {red }}$ is a subgroup scheme, it need not be normal, for example in semidirect products like $G=\alpha_{p} \rtimes \mathbb{G}_{m}$. 
The situation simplifies somewhat for commutative group schemes. Recall that if $G$ is commutative and affine, then there is a maximal multiplicative subgroup scheme $G^{\text {mult }} \subset G$, and the quotient is unipotent ([DG70, Chapter IV, §3, Theorem 1.1]). If the resulting quotient is finite, Lemma 1.3 gives $\Upsilon_{G}=G / G^{\text {mult }}$. In general, we get:

Proposition 1.7. Suppose that $G$ is commutative, that the scheme $G_{\text {red }}^{0}$ is geometrically reduced, and that the projection $G \rightarrow \Phi_{G}$ admits a section. For the local group scheme $L=G^{0} / G_{\text {red }}^{0}$, we get an identification $\Upsilon_{G}^{0}=L / L^{\text {mult }}$.

Proof. The section gives a decomposition of commutative algebraic groups $G=G^{\prime} \oplus G^{\prime \prime}$, where the first factor is connected and the second factor is étale. In turn, we have $\Upsilon_{G}=\Upsilon_{G^{\prime}} \times \Upsilon_{G^{\prime \prime}}$. From the universal property we infer that $\Upsilon_{G^{\prime \prime}}$ is étale, so we may assume from the start that $G$ is connected, and have to show that the projection $G \rightarrow G / G_{\text {red }}=L$ induces an isomorphism $\Upsilon_{G} \rightarrow \Upsilon_{L}=L / L^{\text {mult }}$. Since $G_{\text {red }}$ is geometrically reduced, the inclusion $G_{\text {red }} \subset G$ is a subgroup scheme, which is smooth and connected. It lies in the kernel of any homomorphism $G \rightarrow U$ to some finite unipotent scheme $U$, by Lemma 1.3. From the universal properties we infer that $\Upsilon_{G} \rightarrow \Upsilon_{L}$ is an isomorphism.

This leads to the following structure result:

Theorem 1.8. If $G$ is commutative and $k$ is perfect, we get an identification

$$
\Upsilon_{G}=L / L^{\text {mult }} \times \Phi_{G}\left[p^{\infty}\right],
$$

with the local group scheme $L=G^{0} / G_{\text {red }}^{0}$ and the p-primary part $\Phi_{G}\left[p^{\infty}\right] \subset \Phi_{G}$. Moreover, the kernel $N$ for the projection $G \rightarrow \Upsilon_{G}$ has a three-step filtration with $N / N_{2}$ multiplicative, $N_{2} / N_{1}$ smooth unipotent, and $N_{1}$ anti-affine.

Proof. Since $\Phi_{G}$ is commutative, the quotient of $\Phi_{G}\left(k^{\mathrm{sep}}\right)$ by the subgroup generated by the Sylow-l-groups gets identified with the $p$-primary torsion in $\Phi_{G}\left(k^{\text {sep }}\right)$. From Proposition 1.4 we infer that $\Phi_{G}\left[p^{\infty}\right]$ is the group scheme of components for $\Upsilon_{G}$. Since $k$ is perfect, the scheme $G_{\text {red }}^{0}$ is geometrically reduced, and Proposition 1.7 gives $\Upsilon_{G}^{0}=L / L^{\text {mult }}$. Moreover, the reduced part of $\Upsilon_{G}$ yields a section for the group scheme of components, and we get the decomposition $\Upsilon_{G}=L / L^{\text {mult }} \times \Phi_{G}\left[p^{\infty}\right]$. Applying the Five Lemma to the commutative diagram

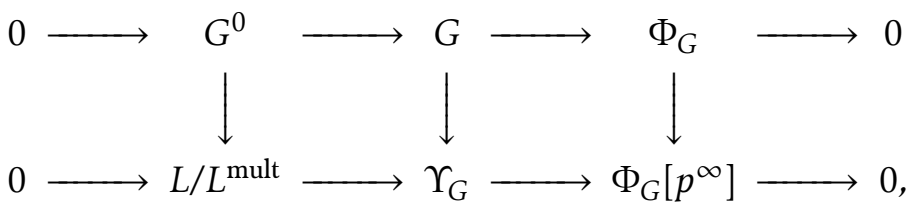

we see that the kernel $N$ for $G \rightarrow \Upsilon_{G}$ has a filtration $F_{i} \subset N$ with

$$
F_{5}=N \quad \text { and } \quad F_{4}=\operatorname{Ker}\left(G^{0} \rightarrow L / L^{\text {mult }}\right) \quad \text { and } \quad F_{3}=\operatorname{Ker}\left(G^{0} \rightarrow L\right)=G_{\text {red }}^{0} .
$$

Then $F_{5} / F_{4}$ is the sum of the $l$-primary parts in $\Phi_{G}$ for the primes $l \neq p$, and $F_{4} / F_{3}=L^{\text {mult }}$. The kernel $F_{1}$ for the affinization $F_{3} \rightarrow F_{3}^{\text {aff }}$ is anti-affine. Moreover, the multiplicative part of $F_{3}^{\text {aff }}$ has a unique complement, because $k$ is perfect, and this complement is smooth unipotent. Let $F_{2} \subset F_{3}$ be its preimage.

Summing up, we have constructed a five-step filtration $0=F_{0} \subset \ldots \subset F_{5}=N$. Setting $N_{2}=F_{2}$ and $N_{1}=F_{1}$ we see that $N / N_{1}$ is multiplicative, $N_{2} / N_{1}$ is smooth unipotent, and $N_{1}$ is anti-affine.

Note that the epimorphism $G \rightarrow \Upsilon_{G}$ does not admit a section in general: suppose that $N$ is either the additive group, or a supersingular elliptic curve. With respect to the scalar multiplication of $\operatorname{End}\left(\alpha_{p}\right)=k$, the abelian group $\operatorname{Ext}^{1}\left(\alpha_{p}, N\right)$ becomes a one-dimensional vector space, provided that $k$ is algebraically closed ([Oor66, table on page II.14-2]). In the ensuing non-split extensions $0 \rightarrow N \rightarrow G \rightarrow \alpha_{p} \rightarrow 0$, the projection coincides with $G \rightarrow \Upsilon_{G}$, according to Lemma 1.3. 


\section{Picard scheme and Bockstein operators}

Let $k$ be a ground field of characteristic $p>0$, and $Y$ be a proper scheme. Then the Picard scheme $P=\operatorname{Pic}_{Y / k}$ exists, and this group scheme is locally of finite type ([SGA6, exposé XII, corollaire 1.5 and exposé XIII, proposition 3.2]). The Galois module for the étale group scheme $\Phi_{P}=P / P^{0}$ is the Néron-Severi group $\Phi_{P}\left(k^{\mathrm{sep}}\right)=\mathrm{NS}\left(Y \otimes_{k} k^{\mathrm{alg}}\right)$, which is finitely generated. In particular, the torsion part in $\Phi_{P}$ is a finite group scheme, hence its inverse image $G=\operatorname{Pic}_{Y / k}^{\tau}$ in the Picard scheme is an algebraic group scheme. We now consider the maximal finite unipotent quotient

$$
\Upsilon_{Y / k}=\Upsilon_{G}=\Upsilon_{\mathrm{Pic}_{Y / k}^{\tau}}
$$

and regard this in many situations as a measure for unusual behavior of torsion in the Picard scheme. From Proposition 1.4 we get:

Proposition 2.1. The Galois module $\Upsilon_{Y / k}\left(k^{\mathrm{sep}}\right)$ is the p-torsion part of the Néron-Severi group $\mathrm{NS}\left(Y \otimes k^{\mathrm{alg}}\right)$.

We now seek to understand the component of the origin $\Upsilon_{Y / k}^{0}$. For this the Frobenius map $f \mapsto f^{p}$ on the structure sheaf $\mathscr{O}_{Y}$ is crucial. This map is additive, and becomes $k$-linear when one re-defines scalar multiplication on the range as $\lambda \cdot f=\lambda^{p} f$. Such additive maps are called $p$-linear. We now consider the induced $p$-linear maps on the cohomology groups $H^{i}\left(Y, \mathscr{O}_{Y}\right)$.

To understand this better, suppose we have an arbitrary finite-dimensional $k$-vector space $V$, together with a $p$-linear map $f: V \rightarrow V$. Choose a basis $a_{1}, \ldots, a_{n} \in V$. Then $f$ is determined by the images $f\left(a_{j}\right)$, and the expansion $f\left(a_{j}\right)=\sum \lambda_{i j} a_{i}$ gives a matrix $A=\left(\lambda_{i j}\right) \in \operatorname{Mat}_{n}(k)$. A different basis $b_{1}, \ldots, b_{n} \in V$ yields another matrix $B=S A T$, where the base-change matrix $S=\left(\sigma_{i j}\right)$ is defined by $a_{j}=\sum \sigma_{i j} b_{i}$, and $T$ is obtained from the inverse of $S$ by applying Frobenius to the entries. In turn, the rank of $A$ depends only on $f$. Let us call this integer the Hasse-Witt rank $\operatorname{rank}_{\mathrm{HW}}(f) \geq 0$ of the $p$-linear map $f: V \rightarrow V$. Note also that one may define the Hasse-Witt determinant $\operatorname{det}_{\mathrm{HW}}(f)=\operatorname{det}(A)$ as a class in the monoid $k / k^{\times(p-1)}$. Furthermore, we may regard the datum $(V, f)$ as a left module over the associative ring $k[F]$, where the relations $F \lambda=\lambda^{p} F$ hold, by setting $F \cdot a=f(a)$. The Hasse-Witt rank and determinant then become invariants of this module. All these considerations go back to Hasse and Witt [HW36], who studied $V=H^{1}\left(C, \mathscr{O}_{C}\right)$ for a smooth algebraic curve $C$ over an algebraically closed field $k$. Compare also the recent discussion of Achter and Howe [AH19] for a discussion of historical developments, and widespread inaccuracies in the literature.

Given a field extension $k \subset k^{\prime}$, we see that there is a unique $p$-linear extension $f^{\prime}$ of $f$ to $V^{\prime}=V \otimes k$, given by $f\left(a_{j} \otimes \lambda\right)=\lambda^{p} f\left(a_{j}\right)$. Obviously, the $p$-linear maps $f$ and $f^{\prime}$ have the same Hasse-Witt rank. If $k$ is perfect, the subgroup $U=f(V)$ inside $V$ is actually a vector subspace with respect to the original scalar multiplication, and we have $\operatorname{rank}_{\mathrm{HW}}(f)=\operatorname{dim}_{k}(U)$. If $\operatorname{rank}_{\mathrm{HW}}(f)=\operatorname{dim}(V)$, we say that $f$ has maximal Hasse-Witt rank. This means that $f(V)$ generates the vector space $V$ with respect to the original scalar multiplication. Equivalently, for some and hence all perfect field extensions $k \subset k^{\prime}$ the $p$-linear extension $f^{\prime}: V^{\prime} \rightarrow V^{\prime}$ is bijective. We now come to the main result of this section:

Theorem 2.2. We have $\Upsilon_{Y / k}^{0}=0$ provided the Frobenius map on the second cohomology group $H^{2}\left(Y, \mathscr{O}_{Y}\right)$ has maximal Hasse-Witt rank.

The proof is deferred to the end of this section. It relies on Bockstein operators, a theory introduced by Serre [Ser58], which we like to discuss first. Write $W_{m}(k)$ be the ring of Witt vectors $\left(\lambda_{0}, \ldots, \lambda_{m-1}\right)$ of length $m$. This is a ring endowed with two commuting additive maps Frobenius $F$ and Verschiebung ("shift") $V$, given by the formula

$$
F\left(\lambda_{0}, \ldots, \lambda_{m-1}\right)=\left(\lambda_{0}^{p}, \ldots, \lambda_{m-1}^{p}\right) \text { and } V\left(\lambda_{0}, \ldots, \lambda_{m-1}\right)=\left(0, \lambda_{0}, \ldots, \lambda_{m-2}\right) .
$$


We refer to [Bou83, chapitre 9, §1] for the general theory of Witt vectors. The canonical projection $W_{m}(k) \rightarrow W_{m-n}(k)$ is a homomorphism of rings, whose kernel we denote by

$$
V_{m}^{n}(k)=V^{m-n} W_{m}(k) .
$$

Note that this kernel has length $m-n$, and is stable under Frobenius, by the relation $F V=V F$. Likewise, we have a short exact sequence of abelian sheaves

$$
0 \longrightarrow V_{m}^{n}\left(\mathscr{O}_{Y}\right) \longrightarrow W_{m}\left(\mathscr{O}_{Y}\right) \longrightarrow W_{m-n}\left(\mathscr{O}_{Y}\right) \longrightarrow 0
$$

where the maps are $W_{m}(k)$-linear and compatible with Frobenius. To simplify notation, write $W_{m}=W_{m}\left(\mathscr{O}_{Y}\right)$ and $V_{m}^{n}=V_{m}^{n}\left(\mathscr{O}_{Y}\right)$, such that $W_{1}=\mathscr{O}_{Y}$. Combining the long exact sequences for the short exact sequences $0 \rightarrow V_{r+1}^{r} \rightarrow W_{r+1} \rightarrow W_{r} \rightarrow 0$ and $0 \rightarrow V_{r}^{1} \rightarrow W_{r} \rightarrow \mathscr{O}_{Y} \rightarrow 0$, we get for each $i \geq 0$ a commutative diagram

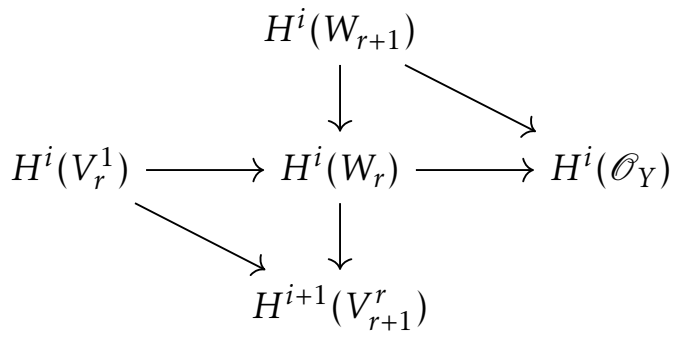

with exact row and column. This gives a canonical $W_{r}(k)$-linear map

$$
\operatorname{Im}\left(H^{i}\left(W_{r}\right) \rightarrow H^{i}\left(\mathscr{O}_{Y}\right)\right) \stackrel{\beta_{r}}{\longrightarrow} \operatorname{Coker}\left(H^{i}\left(V_{r}^{1}\right) \rightarrow H^{i+1}\left(V_{r+1}^{r}\right)\right)
$$

called Bockstein operator, by sending the image of $x \in H^{i}\left(W_{r}\right)$ in $H^{i}\left(\mathscr{O}_{Y}\right)$ to the image of $x$ in $H^{i+1}\left(V_{r+1}^{r}\right)$ modulo the image of $H^{i}\left(V_{r}^{1}\right)$. Obviously, the kernel of $\beta_{r}$ comprises those cohomology classes in $H^{i}\left(\mathscr{O}_{Y}\right)$ that extend to $H^{i}\left(W_{r+1}\right)$. These form a decreasing sequence. We write $H^{i}\left(Y, \mathscr{O}_{Y}\right)[\beta]$ for their common intersection, and call it the Bockstein kernel. By construction, this is a vector subspace of $H^{i}\left(Y, \mathscr{O}_{Y}\right)$ invariant under Frobenius.

Now consider the case $i=1$. Then $\mathfrak{g}=H^{1}\left(Y, \mathscr{O}_{Y}\right)$ is the Lie algebra for the Picard scheme, hence also for the algebraic group scheme $G=\operatorname{Pic}_{Y / k}^{0}$. As such, it has an additional structure, namely the $p$-power map $x \mapsto x^{[p]}$ obtained from the $p$-fold composition of derivations in the associative algebra of differential operators. This turns $\mathfrak{g}$ into a restricted Lie algebra. The interplay between $p$-power map, Lie bracket and scalar multiplication is regulated by three axioms ([DG70, Chapter II, §7]). In our situation, the $p$-power map on the Lie algebra coincides with the Frobenius on cohomology, so the Bockstein kernel $\mathfrak{g}_{\mathrm{red}}=H^{1}\left(Y, \mathscr{O}_{Y}\right)[\beta]$ is a restricted Lie subalgebra. Here we are interested in the Bockstein cokernel $\mathfrak{h}=\mathfrak{g} / \mathfrak{g}_{\text {red }}$, with its inherited structure of restricted Lie algebra.

Recall that for every algebraic group scheme $H$, the relative Frobenius is a homomorphism $F: H \rightarrow H^{(p)}$ of group schemes, and its kernel is a local group scheme $H[F]$. Local group schemes $H$ with $H=H[F]$ are called of height $\leq 1$. According to [DG70, Chapter II, §7, Theorem 4.2], the functor $H \mapsto \operatorname{Lie}(H)$ is an equivalence between the categories of local groups schemes of height $\leq 1$ and finite-dimensional restricted Lie algebras.

Proposition 2.3. Suppose that the Frobenius map on $H^{2}\left(Y, \mathscr{O}_{Y}\right)$ has maximal Hasse-Witt rank. Then the group scheme $H$ of height $\leq 1$ corresponding to the Bockstein cokernel $\mathfrak{h}=H^{1}\left(Y, \mathscr{O}_{Y}\right) / H^{1}\left(Y, \mathscr{O}_{Y}\right)[\beta]$ is multiplicative. If the reduced part of $G=\operatorname{Pic}_{Y / k}^{0}$ is geometrically reduced, then the resulting local group scheme $L=G / G_{\text {red }}$ is also multiplicative.

Proof. It suffices to treat the case that $k$ is perfect. According to Mumford's analysis in [Mum66, Lecture 27], the Lie algebra of the smooth connected group scheme $G_{\text {red }}$ coincides with the Bockstein kernel. In turn, the Bockstein cokernel is the Lie algebra for the local group scheme $L=G / G_{\text {red }}$, which gives an identification 
$H=L[F]$. But $L$ is multiplicative if and only if its Frobenius kernel $H$ is multiplicative, because the higher Frobenius kernels $L\left[F^{i}\right]$ give a filtration on $L$ whose subquotients are isomorphic to $H$.

Seeking a contradiction, we assume that the inclusion $H^{\text {mult }} \subset H$ is not an equality. Since $k$ is perfect, the projection $H \rightarrow H / H^{\text {mult }}$ admits a section, and we get $H=H^{\text {mult }} \oplus U$ for some non-trivial unipotent local group scheme $U$. According to [DG70, Chapter IV, §2, Corollary 2.13], the Lie algebra Lie $(U)$ contains a vector $b \neq 0$ with $b^{[p]}=0$. Choose a vector $a \neq 0$ from $\mathfrak{g}=H^{1}\left(Y, \mathscr{O}_{Y}\right)$ mapping to $b$. Then $a^{[p]} \in \mathfrak{g}_{\text {red }}$.

Now recall that the $p$-power map equals the Frobenius map. By construction $a$ does not lie in the Bockstein kernel. Hence there is a largest integer $r \geq 0$ such that $a$ belongs to the image of $H^{1}\left(Y, W_{r}\right) \rightarrow H^{1}\left(Y, \mathscr{O}_{Y}\right)$. For the Bockstein operator, this means $\beta_{r}(a) \neq 0$. Since $F(a)=a^{[p]}$ lies in the Bockstein kernel, we have $0=\beta_{r}(F(a))=F\left(\beta_{r}(a)\right)$.

By definition, the range of the Bockstein $\beta_{r}$ is a quotient of $H^{2}\left(V_{r+1}^{r}\right)$, and $V_{r+1}^{r}$ consists of tuples $(0, \ldots, 0, \lambda)$. In turn, there is an identification $V_{r+1}^{r}=\mathscr{O}_{Y}$ of abelian sheaves, compatible with Frobenius. By assumption, the Frobenius is bijective on $H^{2}\left(Y, \mathscr{O}_{Y}\right)$. With Lemma 2.4 below we infer that it is also bijective on the the range of the Bockstein operator. This gives $\beta_{r}(a)=0$, contradiction.

In the above arguments, we have used the following simple observation:

Lemma 2.4. Let $V^{\prime} \rightarrow V \rightarrow V^{\prime \prime}$ be an exact sequence of $k[F]$-modules whose underlying $k$-vector spaces are finite-dimensional. If $V^{\prime}$ and $V^{\prime \prime}$ have maximal Hasse-Witt rank, the same holds for $V$.

Proof. It suffices to treat the case that $k$ is perfect. We then have to check that the $p$-linear map $F: V \rightarrow V$ is surjective. Clearly, its image contains the image of $V^{\prime} \rightarrow V$. This reduces us to the case $V^{\prime}=0$, such that $V \subset V^{\prime \prime}$. Choose a vector space basis $a_{1}, \ldots, a_{r} \in V$ and extend it to a basis $a_{1}, \ldots, a_{n} \in V^{\prime \prime}$. Then $F\left(a_{j}\right)=\sum \lambda_{i j} a_{i}$ defines a matrix $A^{\prime \prime}=\left(\lambda_{i j}\right)$. In turn, the Hasse-Witt $\operatorname{determinant} \operatorname{det}\left(A^{\prime \prime}\right)$ is non-zero. Since $V \subset V^{\prime \prime}$ is an $F$-invariant subspace, we see that $\lambda_{i j}=0$ for $1 \leq j \leq r<i$, and conclude that also $F: V \rightarrow V$ has non-zero Hasse-Witt determinant.

Proof of Theorem 2.2. Suppose that the Frobenius has maximal Hasse-Witt rank on $H^{2}\left(Y, \mathscr{O}_{Y}\right)$. We have to check that $\Upsilon_{G}^{0}=0$ for the algebraic group scheme $G=\operatorname{Pic}_{Y / k}^{\tau}$. In light of Proposition 1.2, it suffices to treat the case that $k$ is perfect. Then $G_{\text {red }}^{0}$ is a subgroup scheme, and by Theorem 1.8 we have to verify that the local group scheme $L=G^{0} / G_{\text {red }}^{0}$ is multiplicative. This holds by Proposition 2.3.

\section{The case of surfaces}

We keep the assumptions of the previous section, so that $\Upsilon_{Y / k}$ is the maximal unipotent quotient of $G=\operatorname{Pic}_{Y / k}^{\tau}$, where $Y$ is a proper scheme over our ground field $k$ of characteristic $p>0$. The goal now is to apply the general results of the previous section to certain classes of $Y$, and establish vanishing results. For simplicity, we assume that $Y$ is equi-dimensional, of dimension $n \geq 0$. Our first observation is:

Proposition 3.1. We have $\Upsilon_{Y / k}=0$ provided that $Y$ is a curve.

Proof. The Néron-Severi group NS $\left(X \otimes k^{\text {alg }}\right)$ is a free group, according to [BLR90, Section 9.4, Corollary 14]. Hence by Proposition 2.1, the group scheme of components for $\Upsilon_{Y / k}$ is trivial. Furthermore, the group $H^{2}\left(Y, \mathscr{O}_{Y}\right)$ vanishes by dimension reason, so the Frobenius has a priori maximal Hasse-Witt rank. The the assertion thus follows from Theorem 2.2.

Let $\omega_{Y}$ be the dualizing sheaf, with its trace map $\operatorname{tr}: H^{n}\left(Y, \omega_{Y}\right) \rightarrow k$. In turn, for every coherent sheaf $\mathscr{F}$ we get a pairing

$$
H^{i}(Y, \mathscr{F}) \times \operatorname{Ext}^{n-i}\left(\mathscr{F}, \omega_{Y}\right) \stackrel{\text { can }}{\longrightarrow} H^{n}\left(Y, \omega_{Y}\right) \stackrel{\text { tr }}{\longrightarrow} k,
$$

which is non-degenerate for $i=n$, regardless of the singularities. The pairings remain non-degenerate in all degrees $i \leq n$ provided that $Y$ is Cohen-Macaulay. We record: 
Proposition 3.2. We have $\Upsilon_{Y / k}^{0}=0$ provided that the scheme $Y$ has dimension $n \geq 2$, is Cohen-Macaulay, and has the property $H^{n-2}\left(Y, \omega_{Y}\right)=0$.

Proof. The above Serre Duality gives $h^{2}\left(\mathscr{O}_{Y}\right)=h^{n-2}\left(\omega_{Y}\right)=0$, and the assertion follows from Theorem 2.2 .

Now suppose that $Y$ is a surface. For every invertible sheaf $\mathscr{L}$, the Euler characteristic $\chi\left(\mathscr{L}^{\otimes t}\right)$ is a numerical polynomial of degree two in the variable $t$, which can be written as

$$
\chi\left(\mathscr{L}^{\otimes t}\right)=\frac{(\mathscr{L} \cdot \mathscr{L})}{2} t^{2}-\frac{\left(\mathscr{L} \cdot \omega_{Y}\right)}{2} t+\chi\left(\mathscr{O}_{Y}\right)
$$

The quadratic term is determined by the self-intersection number $(\mathscr{L} \cdot \mathscr{L})$, whereas the linear term defines an integer $\left(\mathscr{L} \cdot \omega_{Y}\right)$, which coincides with the usual intersection number of invertible sheaves provided that $Y$ is Gorenstein.

Theorem 3.3. Let $Y$ be a reduced surface. Suppose there is an invertible sheaf $\mathscr{L}$ such that for each irreducible component $Z \subset Y$, the restriction $\mathscr{L}_{Z}$ is nef and has $\left(\mathscr{L}_{Z} \cdot \omega_{Z}\right)<0$. Then the cohomology group $H^{2}\left(Y, \mathscr{O}_{Y}\right)$ vanishes, the Néron-Severi group $\mathrm{NS}\left(Y \otimes k^{\mathrm{alg}}\right)$ is free, and the maximal unipotent finite quotient $\Upsilon_{Y / k}$ is trivial.

Proof. The statement on the maximal unipotent quotient follows from the assertions on cohomology and Néron-Severi group, using Proposition 2.1 and Theorem 2.2. To proceed, we first reduce to the case of integral surfaces that are Cohen-Macaulay. Let $X_{1}, \ldots, X_{r}$ be the $S_{2}$-ization of the irreducible components $Y_{1}, \ldots, Y_{r} \subset Y$, and write $X$ for their disjoint union (see for example [SV04] for details on the $S_{2}$-ization). The resulting finite morphism $f: X \rightarrow Y$ is surjective, hence induces an injection on Néron-Severi groups. Moreover, the cokernel in the short exact sequence $0 \rightarrow \mathscr{O}_{Y} \rightarrow f_{*}\left(\mathscr{O}_{X}\right) \rightarrow \mathscr{F} \rightarrow 0$ is at most zero-dimensional (see [EGA4-II, corollaire 5.10.15]). In the resulting long exact sequence

$$
H^{1}(Y, \mathscr{F}) \longrightarrow H^{2}\left(Y, \mathscr{O}_{Y}\right) \longrightarrow H^{2}\left(X, \mathscr{O}_{X}\right) \longrightarrow H^{2}(Y, \mathscr{F}),
$$

the outer terms vanish, and we get an identification $H^{2}\left(Y, \mathscr{O}_{Y}\right)=H^{2}\left(X, \mathscr{O}_{X}\right)$. Now fix some irreducible component $Z=Y_{i}$, and consider the resulting connected component $Z^{\prime}=X_{i}$. Its dualizing sheaf is given by $f_{*}\left(\omega_{Z^{\prime}}\right)=\underline{\operatorname{Hom}}\left(f_{*}\left(\mathscr{O}_{Z^{\prime}}\right), \omega_{Z}\right)$, and we get a short exact sequence $0 \rightarrow f_{*}\left(\omega_{Z^{\prime}}\right) \rightarrow \omega_{Z} \rightarrow \mathscr{G} \rightarrow 0$ where the cokernel is at most zero-dimensional. Tensoring with $\mathscr{L}^{\otimes t}$ and comparing linear terms in the numerical polynomials, we see that $\left(\mathscr{L}_{Z^{\prime}} \cdot \omega_{Z^{\prime}}\right)=\left(\mathscr{L}_{Z} \cdot \omega_{Z}\right)$. Clearly, the pullback of $\mathscr{L}$ under the finite surjection $Z^{\prime} \rightarrow Z$ remains nef. Summing up, it suffices to treat the case that $Y$ is integral and Cohen-Macaulay.

Next, we verify that $h^{2}\left(\mathscr{O}_{Y}\right)=0$. Seeking a contradiction, we assume that there is a short exact sequence $0 \rightarrow \mathscr{O}_{Y} \rightarrow \omega_{Y} \rightarrow \mathscr{F} \rightarrow 0$, where $\mathscr{F}$ is a torsion sheaf. Tensoring with $\mathscr{L}^{\otimes t}$ and using Serre Duality, we get

$$
\chi\left(\mathscr{L}^{\otimes t}\right)+\chi\left(\mathscr{L}^{\otimes t} \otimes \mathscr{F}\right)=\chi\left(\mathscr{L}^{\otimes t} \otimes \omega_{Y}\right)=\chi\left(\mathscr{L}^{\otimes-t}\right) .
$$

Let $\zeta_{i} \in Y$ be the generic points of $\operatorname{Supp}(\mathscr{F})$ whose closures $C_{i} \subset Y$ are one-dimensional, write $m_{i} \geq 1$ for the length of the finite $\mathscr{O}_{Y, \zeta_{i}}$-module $\mathscr{F}_{a_{i}}$, and let $C$ be the union $\bigcup C_{i}$. By the results in [Kle66, Section II.2], we have $\chi\left(\mathscr{L}^{\otimes t} \otimes \mathscr{F}\right)=\sum m_{i}\left(\mathscr{L} \cdot C_{i}\right) t+\chi\left(\mathscr{O}_{C}\right)$, with $\sum m_{i}\left(\mathscr{L} \cdot C_{i}\right) \geq 0$. In equation (1), the linear term on the left has coefficient $-\frac{1}{2}\left(\mathscr{L} \cdot \omega_{Y}\right)+\sum m_{i}\left(\mathscr{L} \cdot C_{i}\right)>0$, whereas the coefficient on the right is $\frac{1}{2}\left(\mathscr{L} \cdot \omega_{Y}\right)<0$, contradiction. This shows $h^{2}\left(\mathscr{O}_{Y}\right)=0$.

It remains to verify the assertion on the Néron-Severi group. Let $f: X \rightarrow Y$ be some resolution of singularities. This proper surjective morphism induces an inclusion NS $(Y) \subset \mathrm{NS}(X)$. Furthermore, we obtain a short exact sequence $0 \rightarrow f_{*}\left(\omega_{X}\right) \rightarrow \omega_{Y} \rightarrow \mathscr{F} \rightarrow 0$ for some torsion sheaf $\mathscr{F}$. Tensoring with $\mathscr{L}^{\otimes t}$, and using Serre duality alongside the Leray-Serre spectral sequence, we get

$$
\chi\left(\mathscr{L}^{\otimes-t}\right)=\chi\left(\mathscr{L}_{X}^{\otimes-t}\right)-\chi\left(\mathscr{L}^{\otimes t} \otimes R^{1} f_{*} \mathscr{O}_{X}\right)+\chi\left(\mathscr{L}^{\otimes t} \otimes \mathscr{F}\right) .
$$

Looking at the linear terms and using the notation of the preceding paragraph, we get

$$
\frac{1}{2}\left(\mathscr{L} \cdot \omega_{Y}\right)=\frac{1}{2}\left(\mathscr{L}_{X} \cdot \omega_{X}\right)+\sum m_{i}\left(\mathscr{L} \cdot C_{i}\right),
$$


and conclude that $\left(\mathscr{L}_{X} \cdot \omega_{X}\right)<0$. This reduces us to the case of regular irreducible surfaces $Y$. Likewise, one easily reduces to the case that $k$ is separably closed.

The base-change to the algebraic closure $k^{\text {alg }}$ is not necessarily regular or normal, not even reduced. Let $X \rightarrow Y \otimes k^{\text {alg }}$ be the normalization of the reduction, and consider the composite morphism $f: X \rightarrow Y$. According to a result of Tanaka ([Tan18, Theorem 4.2], see also [PW17, Theorem 1.1]), we have the following equality $\omega_{X}=f^{*}\left(\omega_{Y}\right) \otimes \mathscr{O}_{X}(-R)$ for some curve $R \subset X$. In turn, $\left(\mathscr{L}_{X} \cdot \omega_{X}\right)<0$. Now let $r: S \rightarrow X$ be the minimal resolution of singularities, with exceptional divisor $E=E_{1}+\ldots+E_{r}$, and $K_{S / X}=\sum \lambda_{i} E_{i}$ be the unique $\mathbb{Q}$-divisor with $\left(K_{S / X} \cdot E_{i}\right)=\left(K_{S} \cdot E_{i}\right)$. Since the resolution is minimal, we must have $\lambda_{i} \leq 0$. Now choose some Weil divisor $K_{X}$ representing $\omega_{X}$, and consider the rational pullback $r^{*}\left(K_{X}\right)$ in the sense of Mumford [Mum61], compare also [Sch19]. We then have

$$
\left(\mathscr{L}_{S} \cdot \omega_{S}\right)=f^{*}(\mathscr{L}) \cdot\left(K_{S / X}+f^{*} K_{X}\right)=\left(\mathscr{L}_{X} \cdot \omega_{X}\right)<0 .
$$

Using that $\mathscr{L}_{S}$ is nef, we infer that the plurigenera $h^{0}\left(\omega_{S}^{\otimes n}\right), n \geq 1$ of the smooth surface $S$ vanish, so its Kodaira dimension must be $\operatorname{kod}(S)=-\infty$. By the Enriques classification, the surface is either $S=\mathbb{P}^{2}$ or admits a ruling. In both cases, NS $(S)$ is free. In turn, the same holds for the subgroup $\mathrm{NS}\left(Y \otimes k^{\text {alg }}\right)$.

This applies in particular for reduced del Pezzo surfaces $Y$, which by definition are Gorenstein, with $\omega_{Y}$ anti-ample, and have $h^{0}\left(\mathscr{O}_{Y}\right)=1$.

\section{Corollary 3.4. Let $Y$ be a reduced del Pezzo surface. Then $\Upsilon_{Y / k}$ is trivial.}

Note that Miles Reid [Rei94] has classified reduced non-normal del Pezzo surfaces over algebraically closed fields. Then the algebraic group $G=\operatorname{Pic}_{Y / k}^{\tau}$ is smooth, and there are cases with $H^{1}\left(Y, \mathscr{O}_{Y}\right) \neq 0$.

Tanaka constructed Mori fiber spaces in characteristic $p \leq 3$, where the generic fiber is a normal projective surfaces $Y$ with $h^{0}\left(\mathscr{O}_{Y}\right)=1$ having only $\mathbb{Q}$-factorial klt-terminal singularities, the $\mathbb{Q}$-divisor $K_{Y}$ is anti-ample, yet the Picard group contains elements of order $p$ ([Tan16, Theorem 1.2], with further investigation in [BT20]). From Theorem 3.3, we see that such elements must come from the component $\mathrm{Pic}_{Y / k}^{0}$ of the origin.

Note also that the reducedness assumption in our results is indispensable: Suppose that $S$ is an irreducible smooth surface, and let $\mathscr{L}$ be an invertible sheaf such that its dual is ample. After passing to suitable multiples, we achieve that $h^{1}\left(\mathscr{L}^{\vee}\right)=h^{2}\left(\mathscr{L}^{\vee}\right)=0$, and $\mathscr{L} \otimes \omega_{S}$ becomes anti-ample. Consider the quasicoherent $\mathscr{O}_{S}$-algebra $\mathscr{A}=\mathscr{O}_{Y} \oplus \mathscr{L}^{\vee}$, with multiplication $(f, s) \cdot\left(f^{\prime}, s^{\prime}\right)=\left(f f^{\prime}, f s^{\prime}+f^{\prime} s\right)$, and let $Y=\operatorname{Spec}(\mathscr{A})$ be its relative spectrum. The structure morphism $f: Y \rightarrow S$ has a canonical section, which is given by the projection $\mathscr{A} \rightarrow \mathscr{O}_{Y}$ and identifies $S$ with $Y_{\text {red. }}$. One also says that $Y$ is a ribbon on $S$. The resulting short exact sequence of abelian sheaves $0 \rightarrow \mathscr{L}^{\vee} \rightarrow \mathscr{O}_{Y}^{\times} \rightarrow \mathscr{O}_{S}^{\times} \rightarrow 1$ shows that the inclusion $S \subset Y$ induces an identification of Picard schemes. Moreover, the relative dualizing sheaf for $f: Y \rightarrow S$ is given by $f_{*}\left(\omega_{Y / S}\right)=\underline{\operatorname{Hom}}\left(\mathscr{A}, \mathscr{O}_{Y}\right)=\mathscr{A} \otimes \mathscr{L}$. In turn, we have $\omega_{Y}=f^{*}\left(\mathscr{L} \otimes \omega_{S}\right)$, which is anti-ample. Summing up, $Y$ is an irreducible non-reduced del Pezzo surface with $\Upsilon_{Y}=\Upsilon_{S}$. The latter easily becomes non-trivial, e.g. if $S$ is a simply-connected Enriques surface in characteristic $p=2$.

\section{Enriques surfaces and del Pezzo surfaces}

Let $k$ be an algebraically closed ground field of characteristic $p=2$. In this section, we construct certain normal Enriques surfaces $Z$ where the numerically trivial part of the Picard scheme is unipotent of order two, together with a finite universal homeomorphism $v: Z^{\prime} \rightarrow Z$ of degree two from a normal del Pezzo surface of degree four with Picard number one. Theses surfaces will arise from very special simply-connected Enriques surfaces.

Throughout, $S$ denotes an Enriques surface. This means that $S$ is a regular connected surface with $c_{1}=0$ and $b_{2}=10$. The group $\operatorname{Num}(S)=\operatorname{Pic}(S) / \operatorname{Pic}^{\tau}(S)$ of numerical classes is a free abelian group of rank $\rho=10$ called the Enriques lattice. The intersection form is isomorphic to $E_{8} \oplus H$, comprising the root 
lattice of type $E_{8}$ and the hyperbolic lattice $H=\left(\begin{array}{ll}0 & 1 \\ 1 & 0\end{array}\right)$. The group scheme $P=\operatorname{Pic}_{S / k}^{\tau}$ of numerically trivial invertible sheaves is finite of order two. In characteristic $p=2$, there are three possibilities for $P$, namely

$$
\mu_{2} \text { and } \mathbb{Z} / 2 \mathbb{Z} \text { and } \alpha_{2}
$$

The respective Enriques surfaces $S$ are called ordinary, classical and supersingular. The inclusion $P \subset \operatorname{Pic}_{S / k}$ yields a $G$-torsor $\epsilon: \tilde{S} \rightarrow S$, where $G=\underline{\operatorname{Hom}}\left(P, \mathbb{G}_{m}\right)$ is the Cartier dual. For ordinary $S$ the Cartier dual $G=\mathbb{Z} / 2 \mathbb{Z}$ is étale, and the total space $\tilde{\tilde{S}}$ is a $K 3$ surface. In the other two cases $P$ is unipotent, $G$ is local, and the integral Gorenstein surface $\tilde{S}$ necessarily acquires singularities. One says that $S$ is a simply-connected Enriques surface, and that $\tilde{S}$ is its K3-like covering. Note that the relation between inclusions $P \subset \operatorname{Pic}_{S / k}$ and $G$-torsors $\tilde{S} \rightarrow S$ goes back to Raynaud [Ray70]. The trichotomy of Enriques surfaces for $p=2$ was developed by Bombieri and Mumford [BM76]. For more information on K3-like coverings, see for example [Sch17].

We write $\gamma: S^{\prime} \rightarrow \tilde{S}$ for the normalization. Since $S$ is regular and $S^{\prime}$ and $\tilde{S}$ are Cohen-Macaulay, both projections $v: S^{\prime} \rightarrow S$ and $\epsilon: \tilde{S} \rightarrow S$ are finite and flat of degree two ([EGA4-II, proposition 6.1.5]). Hence all fibers take the form $\operatorname{Spec} \kappa(s)[\epsilon]$, which ensures that both $S^{\prime}$ and $\tilde{S}$ are Gorenstein. The relative dualizing sheaf for $\gamma: S^{\prime} \rightarrow \tilde{S}$ is given by

$$
\gamma_{*}\left(\omega_{S^{\prime} / \tilde{S}}\right)=\underline{\operatorname{Hom}}\left(\gamma_{*}\left(\mathscr{O}_{S^{\prime}}\right), \mathscr{O}_{\tilde{S}}\right) .
$$

It is also the ideal sheaf for the ramification locus $R \subset S^{\prime}$ of the normalization map. This Weil divisor must be Cartier, because $S^{\prime}$ and $\tilde{S}$ are Gorenstein. As explained in [ESB04], there is a unique effective divisor $C \subset S$ with $R=v^{-1}(C)$. Ekedahl and Shepherd-Barron call $C \subset S$ the conductrix of the Enriques surface $S$. Note that $S$ is simply-connected if $C$ is non-empty.

Now suppose that $E=E_{1}+\ldots+E_{r}$ is a configuration of $(-2)$-curves whose intersection matrix $\left(E_{i} \cdot E_{j}\right)$ is negative-definite. We also say that $E$ is an $A D E$-curve. Let $f: S \rightarrow Z$ be its contraction. The resulting surfaces $Z$ are called normal Enriques surfaces. They are projective and their singularities are rational double points, such that $\omega_{Z}=f_{*}\left(\omega_{S}\right)$ and $\omega_{S}=f^{*}\left(\omega_{Z}\right)$. By the Hodge Index Theorem, we have $r \leq 9$.

Proposition 4.1. The numerical group $\operatorname{Num}(Z)$ if free of rank $\rho=10-r$, and the homomorphism of group schemes $f^{*}: \operatorname{Pic}_{Z / k}^{\tau} \rightarrow \operatorname{Pic}_{S / k}^{\tau}$ is an isomorphism.

Proof. The Picard group of $Z$ can be viewed as the orthogonal complement of the curves $E_{1}, \ldots, E_{r} \in \operatorname{Pic}(S)$, via the preimage map $f^{*}: \operatorname{Pic}(Z) \rightarrow \operatorname{Pic}(S)$. It follows that $\operatorname{Pic}(Z)$ is finitely generated of rank $\rho=10-r$, and the assertion on the numerical group $\operatorname{Num}(Z)$ follows.

The subgroup $\operatorname{Pic}^{\tau}(Z) \subset \operatorname{Pic}(Z)$ is the torsion part. This is cyclic of order two provided that $S$ is classical, and then the assertion on $\mathrm{Pic}_{Z / k}^{\tau}$ is already contained in the preceding paragraph. It remains to treat the case that $S$ is ordinary or supersingular. In other words, the group scheme $P=\operatorname{Pic}_{Z / k}^{\tau}$ is local of height one, with one-dimensional tangent space $\operatorname{Lie}(P)=H^{1}\left(S, \mathscr{O}_{S}\right)$. The Leray-Serre spectral sequence for the contraction $f: S \rightarrow Z$ gives an exact sequence

$$
0 \longrightarrow H^{1}\left(Z, \mathscr{O}_{Z}\right) \longrightarrow H^{1}\left(S, \mathscr{O}_{S}\right) \longrightarrow H^{0}\left(Z, R^{1} f_{*}\left(\mathscr{O}_{S}\right)\right)
$$

The term on the right vanishes, because $Z$ has only rational singularities, and it follows that $\operatorname{Pic}_{Z / k} \rightarrow \operatorname{Pic}_{S / k}$ induces a bijection on tangent spaces. This homomorphism of groups schemes must be a monomorphism, because $\mathscr{O}_{S}=f_{*}\left(\mathscr{O}_{X}\right)$. In turn, the inclusion $\operatorname{Pic}_{Z / k}^{\tau} \subset \operatorname{Pic}_{S / k}^{\tau}$ is an isomorphism.

Set $P=\operatorname{Pic}_{S / k}^{\tau}=\operatorname{Pic}_{Z / k}^{\tau}$ and let $G=\underline{\operatorname{Hom}}\left(P, \mathbb{G}_{m}\right)$ be the Cartier dual. The inclusion of $P \subset \operatorname{Pic}_{Z / k}$ corresponds to a $G$-torsor $\tilde{Z} \rightarrow Z$, and the total space $Z^{\prime}$ is an integral Gorenstein surface. Let $Z^{\prime} \rightarrow \tilde{Z}$ be its normalization. Of course, we have analogous constructions for the Enriques surface $S$, and by naturality 
we get a commutative diagram

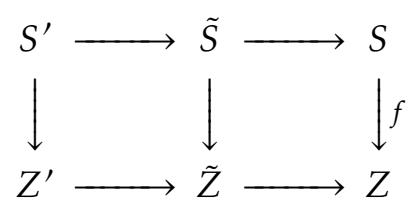

of projective integral surfaces. We write $v: Z^{\prime} \rightarrow Z$ for the composition of the lower arrows. The image $D=f(C)$ is a Weil divisor on the normal surface $Z$. We call it the conductrix of the normal Enriques surface.

Theorem 4.2. Suppose the Enriques surface $S$ and the chosen ADE-curve $E \subset S$ satisfy the following three conditions:

(i) The conductrix $C \subset S$ is not supported by the exceptional divisor $E$.

(ii) There are integers $m_{1}, \ldots, m_{r}$ such that $\left(C \cdot E_{j}\right)=\sum m_{i}\left(E_{i} \cdot E_{j}\right)$ for all $1 \leq j \leq r$.

(iii) The ADE curve $E=E_{1}+\ldots+E_{r}$ has $r=9$ irreducible components.

Then the conductrix $D \subset Z$ is Cartier, and $Z^{\prime}$ is a normal del Pezzo surface of degree $K_{Z^{\prime}}^{2}=2 D^{2}$, with canonical class $K_{Z^{\prime}}=-v^{-1}(D)$, irregularity $h^{1}\left(\mathscr{O}_{Z^{\prime}}\right)=0$ and Picard group $\operatorname{Pic}\left(Z^{\prime}\right)=\mathbb{Z}$. Moreover, all singularities on $Z^{\prime}$ are rational double points.

Proof. Since the horizontal maps in the diagram (2) are universal homeomorphisms and the scheme $Z$ is $\mathbb{Q}$-factorial, the schemes $Z^{\prime}, \tilde{Z}$ and $Z$ have the same Picard number, so Condition (iii) yields $\rho\left(Z^{\prime}\right)=\rho(Z)=1$. Condition (i) ensures that the Weil divisor $D \subset Z$ is non-empty, and (ii) means that it is Cartier.

By definition, the preimage of the conductrix $C \subset S$ on $S^{\prime}$ is the ramification locus for the normalization $S^{\prime} \rightarrow \tilde{S}$. Let $U \subset \tilde{Z}$ be the preimage of the regular locus $\operatorname{Reg}(Z)$. Since $\tilde{S} \rightarrow \tilde{Z}$ becomes an isomorphism over $U$, the preimage of $D$ in $\tilde{Z}$ and the branch locus in $\tilde{Z}$ for the normalization $Z^{\prime} \rightarrow \tilde{Z}$ coincide, at least over $U$. But the preimage $v^{-1}(D)$ and the ramification curve $R \subset Z^{\prime}$ have no embedded components, and $Z^{\prime}$ is normal, so $v^{-1}(D)=R$. As $D \subset Z$ is Cartier, the same holds for $R \subset Z^{\prime}$. Since $\omega_{\tilde{Z}}$ and $\omega_{Z^{\prime} / \tilde{Z}}=\mathscr{O}_{Z^{\prime}}(-R)$ are invertible, the normal surface $Z^{\prime}$ is Gorenstein. We have $-\left(K_{Z^{\prime}} \cdot R\right)=v^{*}(D)^{2}=\operatorname{deg}(v) \cdot D^{2}=2 D^{2}>0$. By the Nakai criterion, $\omega_{Z^{\prime}}$ is anti-ample, thus $Z^{\prime}$ is a normal del Pezzo surface.

Seeking a contradiction, we now assume that $H^{1}\left(Z^{\prime}, \mathscr{O}_{Z^{\prime}}\right) \neq 0$. This is the tangent space to the Picard scheme, so $A=\mathrm{Pic}_{Z^{\prime} / k}^{0}$ is non-zero. The latter is smooth, because the obstruction group $H^{2}\left(Z^{\prime}, \mathscr{O}_{Z^{\prime}}\right) \simeq$ $H^{0}\left(Z^{\prime}, \omega_{Z^{\prime}}\right)$ vanishes, compare [Mum66, Lecture 27]. In turn, the group scheme $A \neq 0$ is an abelian variety, and we conclude that for each prime $l \neq 2$, there is a $\mu_{l}$-torsor $Z^{\prime \prime} \rightarrow Z^{\prime}$ with connected total space. The projection $v: Z^{\prime} \rightarrow Z$ is a universal homeomorphism, so by [SGA1, exposé IX, théorème 4.10], the finite étale Galois covering $Z^{\prime \prime} \rightarrow Z^{\prime}$ is the base-change of some finite étale Galois covering of $Z$. This implies that $\operatorname{Pic}(Z)$ contains an element of order $l$, contradiction. Thus $h^{1}\left(\mathscr{O}_{Z^{\prime}}\right)=0$.

It then follows $\operatorname{Pic}\left(Z^{\prime}\right)=\mathbb{Z}$ by [Sch01, Lemma 2.1]. It remains to check that the singularities on $Z^{\prime}$ are rational double points. Since $Z^{\prime}$ is Gorenstein, the task is to show that they are rational. Seeking a contradiction, we assume that there is at least one non-rational singularity. Consider the minimal resolution of non-rational singularities $r: Y \rightarrow Z^{\prime}$. According to loc. cit., Theorem 2.2, there exists a fibration $\varphi: Y \rightarrow B$ over some curve of genus $g>0$, and $r: Y \rightarrow Z^{\prime}$ is the contraction of some section $E \subset Y$. Set $V=\operatorname{Reg}(Z)$, and fix some prime $l \neq 2$ such that the finitely generated abelian group $\operatorname{Pic}(V)=\operatorname{Pic}(S) / \sum \mathbb{Z} E_{i}$ contains no element of order $l$. As in the preceding paragraph, we find some $\mu_{l}$-torsor $Y^{\prime} \rightarrow Y$, which yields a finite étale Galois covering of $V$. This implies that $\operatorname{Pic}(V)$ contains an element of order $l$, contradiction.

Corollary 4.3. Keep the assumptions of Theorem 4.2. Then the normal del Pezzo surface $Z^{\prime}$ is not regular, we have $\operatorname{Pic}\left(Z^{\prime}\right)=\mathbb{Z} K_{Z^{\prime}}$, and the Cartier divisor $D \subset Z$ has selfintersection $1 \leq D^{2} \leq 4$.

Proof. Suppose $Z^{\prime}$ were regular. It contains no $(-1)$-curves, because $\rho=1$. According to [Doll2, Theorem 8.1.5], the regular del Pezzo surface $Z^{\prime}$ is isomorphic to $\mathbb{P}^{2}$. But $K_{\mathbb{P}^{2}}^{2}=9$ is odd, whereas $K_{Z^{\prime}}^{2}=2 D^{2}$ is even, contradiction. 
Let $r: X \rightarrow Z^{\prime}$ be the minimal resolution of singularities. Then $X$ is a weak del Pezzo surface, which for integral surfaces means that it is Gorenstein and the inverse of the dualizing sheaf is nef and big. Let $Y$ be a minimal model, obtained from a successive contraction $X=X_{0} \rightarrow \ldots \rightarrow X_{n}=Y$ of $(-1)$-curves. Then $Y$ is either the projective plane or a Hirzebruch surface, and in both cases we have $K_{Y}^{2} \leq 9$. This gives $2 D^{2}=K_{Z^{\prime}}^{2}=K_{X}^{2}=K_{Y}^{2}-n \leq 9$, and in turn $1 \leq D^{2} \leq 4$. In particular, the degree $K_{Z^{\prime}}^{2}$ of the normal del Pezzo surface belongs to the set $\{2,4,6,8\}$.

Suppose the canonical class does not generate the Picard group. Then we are in the situation $K_{Z^{\prime}}=2 A$ for some Cartier divisor $A$, and either $A^{2}=1$ or $A^{2}=2$. In case $K_{Z^{\prime}}^{2}=8$, the surface $X$ is the Hirzebruch surface with invariant $e=2$, and $Z^{\prime}$ is obtained by contraction of the (-2)-curve. It then follows that $K_{Z^{\prime}}$ generates the Picard group, contradiction. Now suppose that $K_{Z^{\prime}}^{2}=4$, with intersection numbers $A^{2}=1$ and $A \cdot K_{Z^{\prime}}=-2$. In turn, the Euler characteristic $\chi(\mathscr{L})=A \cdot\left(A-K_{Z^{\prime}}\right) / 2+\chi\left(\mathscr{O}_{Z^{\prime}}\right)$ of the invertible sheaf $\mathscr{L}=\mathscr{O}_{Z^{\prime}}(A)$ is not an integer, contradiction.

For later use, we also record the following vanishing result:

Corollary 4.4. Keep the assumptions of Theorem 4.2. Then $h^{1}\left(\omega_{Z^{\prime}}^{\otimes-i}\right)=0$ for all integers $i$.

Proof. By Serre Duality, it suffices to verify this for $i \geq 0$. Let $r: X \rightarrow Z^{\prime}$ be the minimal resolution of singularities. Since $Z^{\prime}$ has only rational double points, we have $\omega_{X}^{\otimes-i}=r^{*}\left(\omega_{Z^{\prime}}^{\otimes-i}\right)$ and $h^{1}\left(\omega_{X}^{\otimes-i}\right)=h^{1}\left(\omega_{Z^{\prime}}^{\otimes-i}\right)$. The regular surface $X$ is obtained from its minimal model $Y$, which is the projective plane or a Hirzebruch surface, by a sequence of blowing-ups of closed points. It follows that $X$ lifts to characteristic zero. In particular, we may apply Raynaud's vanishing result ([DI87, Corollary 2.8])

$$
H^{1}\left(X, \omega_{X}^{\otimes-i}\right)=H^{1}\left(X, \Omega_{X / k}^{2} \otimes \mathscr{L}\right)=0
$$

for the nef and big invertible sheaf $\mathscr{L}=\omega_{X}^{\otimes-(i+1)}$.

\section{Exceptional Enriques surfaces}

Now suppose that $S$ is a simply-connected Enriques surface whose conductrix takes the form

$$
C=2 C_{1}+3 C_{3}+5 C_{4}+2 C_{2}+4 C_{5}+4 C_{6}+3 C_{7}+3 C_{8}+2 C_{0}+C_{9},
$$

where the ten irreducible components $C_{0}, \ldots, C_{9}$ are $(-2)$-curves with simple normal crossings having the following dual graph $\Gamma$ :

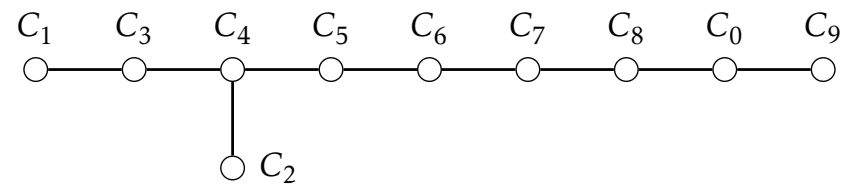

One also says that $S$ is a exceptional Enriques surface of type $T_{2,3,7}$. Here the indices 2,3,7 denote the length of the terminal chains in the star-shaped tree $\Gamma$, including the central vertex $C_{4} \in \Gamma$. Such Enriques surfaces were already considered in the monograph of Cossec and Dolgachev [CD89, Chapter III, §4].

The general notion of exceptional Enriques surfaces was introduced by Ekedahl and Shepherd-Barron [ESB04], who studied them in detail. They can be characterized in terms of the conductrix $C \subset S$, and also by properties of the Hodge ring $\bigoplus_{i j} H^{i}\left(S, \Omega_{S}^{j}\right)$. Explicit equations for birational models were found by Salomonsson [Sal03]. For examples, the equation

$$
z^{2}+\left(y^{4}+x^{4}\right) x^{3} y^{3} s^{4}+\lambda x^{5} y^{3} s^{3} t+x y t=0, \quad \lambda \neq 0,
$$

as well as

$$
z^{2}+x^{3} y^{7} s^{4}+\mu x^{8} s^{3} t+x y t^{4}=0, \quad \mu \neq 0
$$


define birational models for exceptional Enriques surfaces of type $T_{2,3,7}$ as inseparable double covering of a Hirzebruch surface with coordinates $x, y, s, t$. The first equation gives classical, the second equation supersingular Enriques surfaces.

The reduced curve $F=C_{0}+\ldots+C_{8}$ on the Enriques surface $S$ supports a curve of canonical type with Kodaira symbol $\mathrm{II}^{*}$. Let $\varphi: S \rightarrow \mathbb{P}^{1}$ be the resulting genus-one fibrations. This fibration is quasielliptic, and there is no other genus-one fibration, according to [ESB04, Theorem $\mathrm{C}$ ]. The fiber corresponding to $F$ is multiple, because otherwise $2 \leq\left(C_{9} \cdot F\right)=\left(C_{9} \cdot C_{0}\right)=1$, contradiction. Since $b_{2}=10$, all other fibers are irreducible, thus have Kodaira symbol II. If $S$ is classical, there must be another multiple fiber. In the supersingular case, all other fibers are simple ([CD89, Theorem 5.7.2]).

Let $f: S \rightarrow Z$ be the contraction of the ADE-curves $C_{1}+\ldots+C_{8}$ and $C_{9}$. Then $Z$ is a normal Enriques surface with $\operatorname{Sing}(Z)=\{a, b\}$, where the first local ring $\mathscr{O}_{Z, a}$ is a rational double point of type $E_{8}$, and the second local ring $\mathscr{O}_{Z, b}$ is a rational double point of type $A_{1}$. Write $D_{0}=f\left(C_{0}\right)$ for the image of the remaining $(-2)$-curve, which is a Weil divisor. The conductrix of the normal Enriques surface is $D=f(C)=2 D_{0}$. One easily sees that Theorem 4.2 applies, so $D \subset Z$ is Cartier, and we get a normal del Pezzo surface $Z^{\prime}$ as an inseparable double covering $v: Z^{\prime} \rightarrow Z$. The goal of this section is to study the geometry of these surfaces in detail.

Following Hartshorne [Har94], we write $\operatorname{APic}(X)$ for the group of isomorphism classes of reflexive rankone sheaves, on a given normal noetherian scheme $X$. It is called the almost Picard group, and could also be seen as the group of 1-cycles modulo linear equivalence. If $X$ is a proper surface, the group APic $(X)$ is endowed with Mumford's rational selfintersection numbers [Mum61], which extend the usual intersection numbers for invertible sheaves. If $X=\operatorname{Spec}(R)$ is local, we use the more traditional $\mathrm{Cl}(R)=\mathrm{APic}(X)$. In the global case, we prefer $\operatorname{APic}(X)$, because it emphasizes the relation to the Picard group.

Proposition 5.1. The group $\mathrm{APic}(Z)$ is generated by $D_{0}$ and the canonical class $K_{Z}$, with selfintersection number $D_{0}^{2}=1 / 2$. Moreover, the subgroup $\operatorname{Pic}(Z)$ has index two, and is generated by the conductrix $D$, which has $D^{2}=2$, together with $K_{Z}$.

Proof. Via the pullback map $f^{*}: \operatorname{Pic}(Z) \rightarrow \operatorname{Pic}(S)$, we may regard $\operatorname{Pic}(Z)$ as the orthogonal complement of the nine curves $C_{1}, \ldots, C_{9} \in \operatorname{Pic}(S)$. This orthogonal complement has rank one, and is generated by the numerically trivial $K_{Z}$ and the linear combination

$$
2 C_{0}+2\left(2 C_{8}+3 C_{7}+4 C_{6}+5 C_{5}+6 C_{4}+3 C_{2}+2 C_{1}+4 C_{3}\right)+C_{9}
$$

This coincides with $f^{*}(D)$, and gives the selfintersection $D^{2}=f^{*}(D)^{2}=2$. The almost Picard group can be seen as the cokernel for the inclusion $\sum_{i=1}^{9} \mathbb{Z} C_{i} \subset \operatorname{Pic}(S)$. Since $C_{0}, \ldots, C_{9} \in \operatorname{Pic}(S)$ form a basis modulo the torsion part, the assertion on $\operatorname{APic}(Z)$ follows.

Let $\tilde{Z} \rightarrow Z$ be the canonical covering and $Z^{\prime} \rightarrow \tilde{Z}$ be its normalization, as considered in the previous section. Consider the composite morphism $v: Z^{\prime} \rightarrow Z$, which is finite of degree two. According to Theorem 4.2 , the total space $Z^{\prime}$ is a normal del Pezzo surface of degree $K_{Z^{\prime}}^{2}=4$ with $\operatorname{Pic}\left(Z^{\prime}\right)=\mathbb{Z}$. In the next sections, we will embed $Z^{\prime}$ into some normal threefold, and use $v$ as a gluing map for a denormalization. Our goal here is to understand the geometry of the double covering $v: Z^{\prime} \rightarrow Z$. The main task is to understand what happens over the conductrix $D=f(C)$.

The following terminology will be useful: The rational cuspidal curve is the projective scheme Spec $k\left[t^{2}, t^{3}\right] \cup$ Spec $k\left[t^{-1}\right]$, which is the integral singular curve of genus one whose local rings are unibranch. A ribbon on a scheme $X$ is a closed embedding $X \subset Y$ whose ideal sheaf $\mathscr{N} \subset \mathscr{O}_{Y}$ satisfies $\mathscr{N}^{2}=0$, such that the $\mathscr{O}_{Y}$-module $\mathscr{N}$ is actually an $\mathscr{O}_{X}$-module, and that $\mathscr{N}$ is invertible as $\mathscr{O}_{X}$-module. This terminology is due to Bayer and Eisenbud [BE95]. 
Proposition 5.2. The scheme $D_{\text {red }}$ is the rational cuspidal curve, and the conductrix $D=f(C)$ is a ribbon on $D_{\text {red }}$ with ideal sheaf $\mathscr{N}$ of degree $\operatorname{deg}(\mathscr{N})=-1$. The cuspidal point of $D_{\text {red }}$ is located at the $E_{8}$-singularity $a \in Z$.

Proof. The morphism $f: S \rightarrow Z$ factors over the contraction $g: S \rightarrow \bar{S}$ of the ADE-curve $C_{1}+\ldots+C_{8}$. This creates a rational double point $\bar{a} \in \bar{S}$ of type $E_{8}$, and we have $\mathscr{O}_{\bar{S}, \bar{a}}=\mathscr{O}_{Z, a}$. Since the normal surface $\bar{S}$ is locally factorial, the integral curve $\bar{D}_{0}=g\left(C_{0}\right)$ remains Cartier. It contains $\bar{a}$, and the local ring $\mathscr{O}_{\bar{S}, \bar{a}}$ is singular, whence $\mathscr{O}_{\bar{D}_{0}, \bar{a}}$ is singular as well. This singularity on $\bar{D}_{0}$ must be unibranch, in light of the dual graph (3).

Since the fibration $\varphi: S \rightarrow \mathbb{P}^{1}$ factors over $\bar{S}$, we must have $\left(\bar{D}_{0} \cdot \bar{D}_{0}\right)=0$. The Adjunction Formula gives $\operatorname{deg}\left(\omega_{\bar{D}_{0}}\right)=\left(K_{\bar{S}}+\bar{D}_{0}\right) \cdot \bar{D}_{0}=0$. We have $h^{0}\left(\mathscr{O}_{\bar{D}_{0}}\right)=1$, because the curve is integral, hence $h^{1}\left(\mathscr{O}_{\bar{D}_{0}}\right)=1$. The classification of integral curves of genus one shows that $\bar{D}_{0}$ is the rational cuspidal curve.

The induced morphism $h: \bar{S} \rightarrow Z$ is the contraction of the $(-2)$-curve $\bar{C}_{9}$ corresponding to $C_{9} \subset S$, resulting in the rational double point $b \in Z$ of type $A_{1}$. We have $\operatorname{Spec}(k)=\bar{D}_{0} \cap \bar{C}_{9}=\bar{D}_{0} \cap h^{-1}(b)$, the latter by [Art66, Theorem 4]. According to the Nakayama Lemma, the induced morphism $h: \bar{D}_{0} \rightarrow D_{0}$ is an isomorphism. In turn, $D_{\text {red }}=D_{0}$ is the rational cuspidal curve.

The Adjunction Formula for $D \subset Z$ yields $\operatorname{deg}\left(\omega_{D}\right)=\left(K_{Z}+D\right) \cdot D=2$, thus $\chi\left(\mathscr{O}_{D}\right)=-1$. The normal surface $Z$ satisfies Serre's Condition $\left(S_{2}\right)$, so the Cartier divisor $D$ satisfies $\left(S_{1}\right)$. Consequently, the ideal sheaf $\mathscr{N} \subset \mathscr{O}_{D}$ for the closed subscheme $D_{0} \subset D$ is torsion-free. It is invertible at $a \in D_{0}$, where $D_{0}$ is Cartier, and has rank one, hence $\mathscr{N}$ is invertible as sheaf on $D_{0}$. Thus $D$ is a ribbon on the rational cuspidal curve $D_{0}$. The short exact sequence $0 \rightarrow \mathscr{N} \rightarrow \mathscr{O}_{D} \rightarrow \mathscr{O}_{D_{0}} \rightarrow 0$ yields $\chi(\mathscr{N})=\chi\left(\mathscr{O}_{D}\right)-\chi\left(\mathscr{O}_{D_{0}}\right)=-1$. In turn, $\operatorname{deg}(\mathscr{N})=-1$.

Now consider the preimage $D^{\prime}=v^{-1}(D)$ of the conductrix $D \subset Z$ on the normal del Pezzo surface with respect to the double covering $v: Z^{\prime} \rightarrow Z$.

Proposition 5.3. The scheme $D^{\prime}$ is a ribbon on $D_{\text {red }}^{\prime}=\mathbb{P}^{1}$, for the invertible sheaf $\mathscr{M}=\mathscr{O}_{\mathbb{P}^{1}}(-2)$. The morphism $v: D_{\text {red }}^{\prime} \rightarrow D_{\text {red }}$ factors as the normalization map $\mathbb{P}^{1} \rightarrow D_{\text {red }}$ followed by the relative Frobenius $F: \mathbb{P}^{1} \rightarrow \mathbb{P}^{1}$. Moreover, the induced map

$$
\mathscr{O}_{\mathbb{P}^{1}}(-1)^{\otimes 2}=F^{*}\left(\mathscr{O}_{\mathbb{P}^{1}}(-1)\right)=v^{*}(\mathscr{N}) \longrightarrow \mathscr{M}=\mathscr{O}_{\mathbb{P}^{1}}(-2)
$$

\section{is bijective.}

Proof. Let $\zeta \in D$ be the generic point. Then $\mathscr{O}_{D, \zeta}=F[\epsilon]$, where $F=\kappa(\zeta)$ is the function field of $D_{\text {red }}$ and $\epsilon$ is an indeterminate subject to $\epsilon^{2}=0$. The induced extension $F \subset \mathscr{O}_{D^{\prime}, \zeta} /(\epsilon)$ has degree two. Since $Z^{\prime}$ is normal, the local ring $\mathscr{O}_{Z^{\prime}, \zeta}$ is a discrete valuation ring, and the fiber $v^{-1}(\zeta)$ has embedding dimension at most one. It follows that the local Artin ring $\mathscr{O}_{D^{\prime}, \zeta} /(\epsilon)$ is a field, which must be purely inseparable over $F$. This shows that $D^{\prime}=2 D_{\text {red }}^{\prime}$.

The short exact sequence $0 \rightarrow \mathscr{O}_{Z^{\prime}}\left(-D_{\text {red }}^{\prime}\right) \rightarrow \mathscr{O}_{Z^{\prime}} \rightarrow \mathscr{O}_{D_{\text {red }}^{\prime}} \rightarrow 0$ yields an exact sequence

$$
H^{1}\left(Z^{\prime}, \mathscr{O}_{Z^{\prime}}\right) \longrightarrow H^{1}\left(D_{\text {red }}^{\prime}, \mathscr{O}_{D_{\text {red }}^{\prime}}\right) \longrightarrow H^{2}\left(Z^{\prime}, \mathscr{O}_{Z^{\prime}}\left(-D_{\text {red }}^{\prime}\right)\right)
$$

According to Theorem 4.2, the term on the left vanishes, whereas the term on the right is Serre dual to $H^{0}\left(Z^{\prime}, \mathscr{O}_{Z^{\prime}}\left(-D_{\text {red }}^{\prime}\right)\right)$, which vanishes as well. Thus $h^{1}\left(\mathscr{O}_{D_{\text {red }}^{\prime}}\right)=0$, and it follows that $D_{\text {red }}^{\prime}=\mathbb{P}^{1}$.

The Adjunction Formula gives $\operatorname{deg}\left(\omega_{D^{\prime}}\right)=\left(D^{\prime}-D^{\prime}\right) \cdot D^{\prime}=0$, and this implies $\chi\left(\mathscr{O}_{D^{\prime}}\right)=0$. The ideal sheaf $\mathscr{M}$ for the inclusion $D_{\text {red }}^{\prime} \subset D$ has $\chi(\mathscr{M})=\chi\left(\mathscr{O}_{D^{\prime}}\right)-\chi\left(\mathscr{O}_{D_{\text {red }}^{\prime}}\right)=-1$. Furthermore, it is torsion-free and of rank one as sheaf on $D_{\text {red }}^{\prime}=\mathbb{P}^{1}$, thus $\mathscr{M}=\mathscr{O}_{\mathbb{P}^{1}}(-2)$. In turn, $D^{\prime}=v^{-1}(D)$ is a ribbon on $\mathbb{P}^{1}$ with respect to the dualizing sheaf $\omega_{\mathbb{P}^{1}}=\mathscr{O}_{\mathbb{P}^{1}}(-2)$. The morphism $v: Z^{\prime} \rightarrow Z$ induces the map (4), which must be injective. It is bijective, because both sides have the same Euler characteristic. 
Note that a similar situation already occurred in the study of Beauville's Kummer varieties in characteristic two ([Sch09, Proposition 7.3]). We now clarify the flatness properties of the normal del Pezzo surface over the normal Enriques surface:

Corollary 5.4. The double covering $v: Z^{\prime} \rightarrow Z$ is flat precisely over the complement of the $E_{8}$-singularity $a \in Z$.

Proof. According to [Har77, Chapter III, Theorem 9.9], our finite morphism is flat at all points $z \in Z$ where the fiber $v^{-1}(z)$ has length two. Since $Z^{\prime}$ is Cohen-Macaulay, this automatically holds when the local ring $\mathscr{O}_{Z, z}$ is regular ([EGA4-II, proposition 6.1.5]), that is, for $z \neq a, b$. In light of our description of the induced map $v: D_{\text {red }}^{\prime} \rightarrow D_{\text {red }}$ as a composition of relative Frobenius with the normalization, this fiber over the $E_{8}$-singularity $a \in Z$ has length four, whereas the fiber of the $A_{1}$-singularity $b \in Z$ has length two.

It remains to determine the singularities on $Z^{\prime}$, and then to compute the group $\operatorname{APic}\left(Z^{\prime}\right)$. Recall that the normal Enriques surface $Z$ contains a rational double point $b \in Z$ of type $A_{1}$. Let $b^{\prime} \in Z^{\prime}$ be the corresponding point on the normal del Pezzo surface. Around this point, the double covering $v: Z^{\prime} \rightarrow Z$ is flat, so the local ring $\mathscr{O}_{Z^{\prime}, b^{\prime}}$ must be singular. Moreover, all singularities must be rational double points, according to Theorem 4.2. We now make a preliminary observation:

Lemma 5.5. The point $b^{\prime} \in Z^{\prime}$ is the only singularity on the normal del Pezzo surface $Z^{\prime}$ that lies on the preimage $v^{-1}(D)$ of the conductrix. If the Enriques surface $S$ is supersingular, we actually have $\operatorname{Sing}\left(Z^{\prime}\right)=\left\{b^{\prime}\right\}$.

Proof. The reduction $D_{0} \subset Z$ of the conductrix is Cartier away from the singularity $b \in Z$, because the other singularity is factorial. In turn, its preimage $v^{-1}\left(D_{0}\right) \subset Z^{\prime}$ is Cartier away from $b^{\prime} \in Z^{\prime}$. According to Proposition 5.3, this preimage is regular, hence the local rings $\mathscr{O}_{Z^{\prime}, x}$ are regular when $x \in Z^{\prime}$ maps to $D \backslash\{b\}$.

For the second statement, consider the unique genus-one fibration $\varphi: S \rightarrow \mathbb{P}^{1}$, and the normalization $S^{\prime} \rightarrow S$ of the K3-like covering. The arguments from [Sch17, Proposition 8.1] show that the Stein factorization of the composite map $S^{\prime} \rightarrow \mathbb{P}^{1}$ is given by the relative Frobenius map $F: \mathbb{P}^{1} \rightarrow \mathbb{P}^{1}$. In particular, $S^{\prime}$ is regular over the relative smooth locus $\operatorname{Reg}\left(S / \mathbb{P}^{1}\right)$. Now suppose that $S$ is supersingular. Then there is only one multiple fiber, whose reduction is $C_{0}+\ldots+C_{8}$, with Kodaira symbol $\mathrm{II}^{*}$. All other geometric fibers are rational cuspidal curves, and $C_{9} \subset S$ is the curve of cusps. Since $C_{1}+\ldots+C_{8}$ and $C_{9}$ are contracted by $f: S \rightarrow Z$, we see that the singular locus of $Z^{\prime}$ lies over $D_{0}=f\left(C_{0}\right)$.

We now can unravel the picture completely:

Proposition 5.6. The rational double point $\mathscr{O}_{Z^{\prime}, b^{\prime}}$ has type $D_{5}$, and this is the only singularity on the normal del Pezzo surface $Z^{\prime}$.

Proof. Let $r: X \rightarrow Z^{\prime}$ be the minimal resolution of singularities. Then $X$ is a weak del Pezzo surface of degree $K_{X}^{2}=K_{Z^{\prime}}^{2}=4$. It is obtained from $\mathbb{P}^{2}$ by blowing-up $5=9-4$ points. The Picard number is $\rho(X)=6=1+5$, so the morphism $r: X \rightarrow Z^{\prime}$ contracts five $(-2)$-curves $E_{1}, \ldots, E_{5} \subset X$. The possible configurations of rational double points on normal del Pezzo surfaces of degree four were classified by Dolgachev [Dol12, Section 8.3.6]. There are only two possibilities with five exceptional divisors, namely $D_{5}$ or the configuration $A_{3}+A_{1}+A_{1}$.

Seeking a contradiction, we assume that $\operatorname{Sing}\left(Z^{\prime}\right)$ is given by $A_{3}+A_{1}+A_{1}$. In light of Lemma 5.5 , the Enriques surface $S$ must be classical. The unique genus-one fibration $\varphi: S \rightarrow \mathbb{P}^{1}$ thus has two multiple fibers. Without restriction, these occur over the points $0, \infty \in \mathbb{P}^{1}$, with respective Kodaira symbols II $^{*}$ and II. Write $C_{\infty}$ for the reduced part of the multiple fiber $\varphi^{-1}(\infty)$, and $D_{\infty}=f\left(C_{\infty}\right)$ for its image on the normal Enrique surface $Z$. This is the rational cuspidal curve with self-intersection $D_{\infty}^{2}=1 / 2$. Its preimage on the canonical covering $\tilde{Z} \rightarrow Z$, which coincides with the canonical covering for the inclusion $\alpha_{2} \subset \mathrm{Pic}_{D_{\infty} / k}$, must be a ribbon on the projective line, according to [Sch17, Lemma 4.2]. In turn, the preimage on the normal del Pezzo surface is of the form $v^{-1}\left(D_{\infty}\right)=2 \Theta$, where $\Theta=\mathbb{P}^{1}$ and rational selfintersection $\Theta^{2}=1 / 4$. 
Moreover, the Weil divisor $v^{-1}\left(D_{0}\right)=\mathbb{P}^{1}$ is linearly equivalent to $v^{-1}\left(D_{\infty}\right)=2 \Theta$. Since both pass through the singular point $b^{\prime} \in Z^{\prime}$, the local class group $\mathrm{Cl}\left(\mathscr{O}_{Z^{\prime}, b^{\prime}}\right)$ is not annihilated by two, and we conclude that $b^{\prime} \in Z^{\prime}$ has type $A_{3}$ rather than $A_{1}$. Furthermore, the germ $\Theta_{b^{\prime}}$ generates the local class group. The five exceptional curves $E_{i}$ and the strict transform $\Theta^{*} \subset X$ of $\Theta \subset Z^{\prime}$ have normal crossings, because the scheme $\Theta$ is regular. After reordering, their dual graph takes this form:

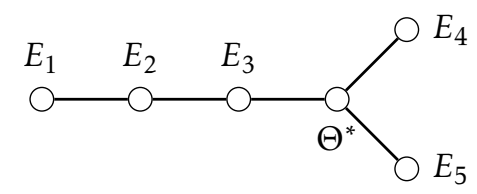

It follows that the rational pull-back in the sense of Mumford [Mum61] is given by

$$
r^{*}(\Theta)=\Theta^{*}+\frac{1}{4}\left(E_{1}+2 E_{2}+3 E_{3}+2 E_{4}+2 E_{5}\right)
$$

which yields $1 / 4=\Theta^{2}=r^{*}(\Theta)^{2}=\left(\Theta^{*} \cdot \Theta^{*}\right)+3 / 4+2 / 4+2 / 4$. Consequently $\left(\Theta^{*} \cdot \Theta^{*}\right)=-6 / 4$, contradicting that this selfintersection number on the regular surface $X$ is an integer.

Proposition 5.7. The normal del Pezzo surface has $\mathrm{APic}\left(Z^{\prime}\right)=\mathbb{Z}$, and the subgroup $\operatorname{Pic}(Z)=Z_{K_{Z^{\prime}}}$ has index four. In particular, the generator $\Theta \in \mathrm{APic}\left(Z^{\prime}\right)$ has selfintersection $\Theta^{2}=1 / 4$.

Proof. Let $r: X \rightarrow Z^{\prime}$ be the minimal resolution of singularities, and $E_{1}, \ldots, E_{5} \subset X$ the five exceptional curves over the rational double point $b^{\prime} \in Z^{\prime}$ of type $D_{5}$, and set $\operatorname{Div}_{E}(X)=\bigoplus \mathbb{Z} E_{i}$. Consider the commutative diagram

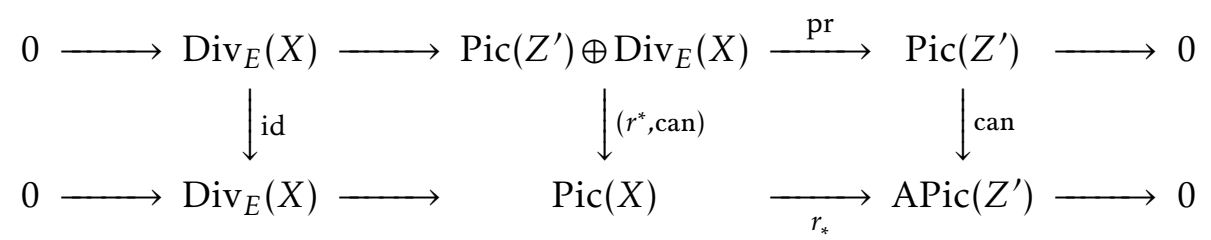

The vertical maps are injective, and the one in the middle has index four, because the intersection forms on $\operatorname{Pic}(X)$ and $\operatorname{Pic}\left(Z^{\prime}\right) \oplus \operatorname{Div}_{E}(X)$ have discriminants $\delta=1$ and $\delta=4^{2}$, respectively. Applying the Snake Lemma, we see that the cokernel for $\operatorname{Pic}\left(Z^{\prime}\right) \subset \operatorname{APic}\left(Z^{\prime}\right)$ has order four. It also sits inside $\operatorname{Cl}\left(\mathscr{O}_{Z^{\prime}, b^{\prime}}\right)$, which is cyclic of order four.

Thus the group $\operatorname{APic}\left(Z^{\prime}\right)$ is an extension of $\mathbb{Z} / 4 \mathbb{Z}$ by $\mathbb{Z}$. It remains to check that it is torsion-free, in other words the inclusion of $L=\operatorname{Div}_{E}(X)$ inside $\operatorname{Pic}(X)$ is primitive. Consider the dual lattice $L^{*}=\operatorname{Hom}(L, \mathbb{Z})$ and the resulting discriminant group $L^{*} / L$, which comes with a perfect $\mathbb{Q} / \mathbb{Z}$-valued pairing. The over-lattices $L \subset L^{\prime}$ correspond to totally isotropic subgroup $T \subset L^{*} / L$, via $T=L^{\prime} / L$, according to [Nik80, Section 4]. In our case, the discriminant group $L^{*} / L=\mathbb{Z} / 4 \mathbb{Z}$ is cyclic, so there are no such subgroups. In turn, $L \subset \operatorname{Pic}(X)$ is primitive, thus $\operatorname{APic}\left(Z^{\prime}\right)=\mathbb{Z}$. The generator $\Theta$ satisfies $4 \Theta=K_{Z^{\prime}}$, and the intersection number $\Theta^{2}=1 / 4$ follows.

By Artin's classification [Art77] of rational double points in positive characteristics, there are actually two isomorphism classes of type $D_{5}$, which are denoted by $D_{5}^{0}$ and $D_{5}^{1}$. The former is simply-connected, the latter not. Since $v: Z^{\prime} \rightarrow Z$ is a finite universal homeomorphism and rational double points of type $A_{1}$ are simply-connected, we see that our singularity $\mathscr{O}_{Z^{\prime}, b^{\prime}}$ is also simply-connected, whence formally given by the equation $z^{2}+y^{2} z+x^{2} y=0$ 


\section{Cones and Fano varieties}

In this section we collect some facts on cones, which complement the discussions by Grothendieck [EGA2, $\S 8]$ and Kollár [Kol13, Section 3.1]. They will be used to construct Fano threefolds with unusual torsion in the next section.

Let $k$ be a ground field of arbitrary characteristic $p \geq 0$ and $B$ be a proper connected scheme. Suppose that $\mathscr{E}$ is a locally free sheaf of rank two, and consider its projectivization

$$
P=\mathbb{P}(\mathscr{E})=\operatorname{Proj}\left(\operatorname{Sym}^{\bullet} \mathscr{E}\right) .
$$

Let $f: P \rightarrow B$ the structure morphism, whose fibers are copies of the projective line $\mathbb{P}^{1}$. From the Formal Function Theorem, one gets a split exact sequence

$$
0 \longrightarrow \operatorname{Pic}(B) \stackrel{f^{*}}{\longrightarrow} \operatorname{Pic}(P) \stackrel{\text { deg }}{\longrightarrow} \mathbb{Z} \longrightarrow 0,
$$

where the degree is taken fiber-wise, and the splitting is given by the tautological sheaf $\mathscr{O}_{P}(1)$. The sections $D \subset P$ correspond to invertible quotients $\mathscr{L}=\mathscr{E} / \mathscr{N}$, via $D=\mathbb{P}(\mathscr{L})$ and $\mathscr{L}=f_{*}\left(\mathscr{O}_{P}(1) \mid D\right)$. Each section is an effective Cartier divisor. Moreover, to simplify the notation, for any line bundle $\mathscr{L}$ on $B$ and any section $D$ in $P$, we denote the restriction $f^{*}(\mathscr{L}) \mid D$ by the same symbol $\mathscr{L}$.

Lemma 6.1. With the above notation, $\mathscr{O}_{P}(D) \simeq f^{*}\left(\mathscr{N}^{\otimes-1}\right) \otimes \mathscr{O}_{P}(1)$. In particular, $\mathscr{O}_{D}(D)=\mathscr{N}^{\otimes-1} \otimes \mathscr{L}$.

Proof. Set $\mathscr{F}=\mathscr{O}_{P}(1) \otimes \mathscr{O}_{P}(-D)$. Tensoring $0 \rightarrow \mathscr{O}_{P}(-D) \rightarrow \mathscr{O}_{P} \rightarrow \mathscr{O}_{D} \rightarrow 0$ with $\mathscr{O}_{P}(1)$ and taking direct images gives $0 \rightarrow f_{*}(\mathscr{F}) \rightarrow \mathscr{E} \rightarrow \mathscr{L} \rightarrow 0$. The map on the right is the quotient map $\mathscr{E} \rightarrow \mathscr{L}$ defining the section $D \subset P$, hence $f_{*}(\mathscr{F})=\mathscr{N}$. The invertible sheaf $\mathscr{F}$ has degree zero on each fiber of $f: P \rightarrow P$. Hence $\mathscr{F}=f^{*}\left(\mathscr{N}^{\prime}\right)$ for some invertible sheaf $\mathscr{N}^{\prime}$ on $B$, by the exact sequence (5). Finally, the Projection Formula gives $\mathscr{N}^{\prime}=f_{*} f^{*}\left(\mathscr{N}^{\prime}\right)=\mathscr{N}$.

From now on, we assume that $\mathscr{E}=\mathscr{O}_{B} \oplus \mathscr{L}$ for some ample invertible sheaf $\mathscr{L}$. Then $P=\mathbb{P}(\mathscr{E})$ contains two canonical sections, namely $A=\mathbb{P}\left(\mathscr{O}_{X}\right)$ and $E=\mathbb{P}(\mathscr{L})$, coming from the two projections $\mathscr{E} \rightarrow \mathscr{O}_{X}$ and $\mathscr{E} \rightarrow \mathscr{L}$, and we have

$$
\mathscr{O}_{A}(A)=\mathscr{L} \quad \text { and } \quad \mathscr{O}_{E}(E)=\mathscr{L}^{\otimes-1} .
$$

Therefore we say that $E \subset S$ is the negative section and $A \subset P$ is the positive section. Clearly, the stable base locus for the invertible sheaf $\mathscr{O}_{P}(A)$ is contained in $A$, and the restriction $\mathscr{O}_{A}(A)$ is ample. According to Fujita's result (see [Fuj83], compare also [Ein00]), the invertible sheaf $\mathscr{O}_{P}(A)$ is semiample, and we get a contraction

$$
r: P \longrightarrow X=\operatorname{Proj} \bigoplus_{i \geq 0} H^{0}\left(P, \mathscr{O}_{P}(i A)\right)
$$

to some projective scheme $X$, with $\mathscr{O}_{P}(n A)=r^{*} \mathscr{O}_{X}(n)$ for some $n \geq 0$ sufficiently large, and $\mathscr{O}_{X}=r_{*}\left(\mathscr{O}_{P}\right)$. Clearly, the connected closed set $E \subset P$ is mapped to a closed point $x_{0} \in X$. The exceptional set for the morphism $r: P \rightarrow X$ is defined as the closed set $\operatorname{Exc}(P / X)=\operatorname{Supp}\left(\Omega_{P / X}^{1}\right)$.

Lemma 6.2. The exceptional set $\operatorname{Exc}(P / X)$ for the morphism $r: P \rightarrow X$ coincides with the negative section $E=\mathbb{P}(\mathscr{L})$.

Proof. The exceptional set is the union of all irreducible curves $C \subset P$ that are disjoint from the positive section $A=\mathbb{P}\left(\mathscr{O}_{B}\right)$. We have to check that each such $C$ is contained in the negative section $E=\mathbb{P}(\mathscr{L})$. For this, we may pass to the base-change $C^{\prime} \times_{B} P$, where $C^{\prime} \rightarrow C$ is the normalization, and assume that $B$ is an irreducible regular curve and $C \subset P$ is a section. Then $P$ is a regular surface, and both $E, C \subset P$ are mapped to points in $X$. Recall that $E \subset P$ corresponds to the invertible quotient $\mathscr{L}=\mathscr{E} / \mathscr{N}$, where $\mathscr{N}=\mathscr{O}_{B}$. By the Hodge Index Theorem, we have $\operatorname{deg}(\mathscr{L})-\operatorname{deg}(\mathscr{N})=E^{2}<0$, and the same holds for $C \subset P$. Since such an invertible quotient $\mathscr{L}=\mathscr{E} / \mathscr{N}$ is unique, $C=E$ follows. 
In turn, the morphism $r: P \backslash E \rightarrow X \backslash\left\{x_{0}\right\}$ is an isomorphism. One also says that $X$ is the projective cone on $B$ with respect to the ample sheaf $\mathscr{L}$, with vertex $x_{0} \in X$. By abuse of notation, we regard the positive section $A=\mathbb{P}\left(\mathscr{O}_{B}\right)$ of the $\mathbb{P}^{1}$-bundles $P=\mathbb{P}(\mathscr{E})$ also as an ample Cartier divisor $A \subset X$.

Proposition 6.3. The projective scheme $X$ has Picard scheme $\operatorname{Pic}_{X / k}=\mathbb{Z}$, and this is generated by the ample sheaf $\mathscr{O}_{X}(A)$.

Proof. Let $\mathscr{F}$ be an invertible sheaf on $X$. Its preimage takes the form $r^{*}(\mathscr{F})=f^{*}(\mathscr{N}) \otimes \mathscr{O}_{P}(d A)$ for some unique $\mathscr{N} \in \operatorname{Pic}(B)$ and $d \in \mathbb{Z}$. Since $r^{*}(\mathscr{F}) \mid E=\mathscr{O}_{E}$ and $\mathscr{O}_{E}(d A)=\mathscr{O}_{E}$, we have $\mathscr{N}=\mathscr{O}_{D}$, and hence $\operatorname{Pic}(X)=\mathbb{Z}$, generated by $\mathscr{O}_{P}(A)$. The statement on the Picard scheme follows likewise, by working with the infinitesimal extension $B \otimes_{k} k[\epsilon]$.

Proposition 6.4. If B is Gorenstein, then P is Gorenstein, and we have the Canonical Bundle Formula

$$
\omega_{P}=\mathscr{O}_{P}(-2 E) \otimes f^{*}\left(\omega_{B} \otimes \mathscr{L}^{\otimes-1}\right) .
$$

Proof. The scheme $P$ must be Gorenstein by [WITO69]. In light of the exact sequence (5), the dualizing sheaf has the form $\omega_{P}=\mathscr{O}_{P}(-2 E) \otimes f^{*}\left(\omega_{B} \otimes \mathscr{N}\right)$ for some invertible sheaf $\mathscr{N}$ on $B$. Since $\mathscr{O}_{E}(E)=\mathscr{L}^{\otimes-1}$, the Adjunction Formula for the effective Cartier divisor $E \subset P$ yields

$$
\omega_{B}=\left(\omega_{P} \otimes \mathscr{O}_{P}(E)\right) \mid E=\mathscr{L}^{\otimes 2} \otimes \omega_{B} \otimes \mathscr{N} \otimes \mathscr{L}^{\otimes-1} .
$$

The assertion follows.

Recall that a scheme $V$ is called a Fano variety if it is proper, with $h^{0}\left(\mathscr{O}_{V}\right)=1$, all local rings $\mathscr{O}_{V, a}$ are Gorenstein, and the dualizing sheaf $\omega_{V}$ is anti-ample. Note that we make no other assumptions on the singularities. Fano varieties of dimension $n \geq 1$ come with two important numerical invariants: the degree and the index

$$
\operatorname{deg}(V)=\left(-K_{V}\right)^{n}=c_{1}^{n}\left(\omega_{V}^{\otimes-1}\right)>0 \quad \text { and } \quad \operatorname{ind}(V)>0 .
$$

The latter is defined as the divisibility of $\omega_{V}$ in the numerical group $\operatorname{Num}(V)$.

Theorem 6.5. Suppose the following:

(i) The projective scheme $B$ is a Fano variety of dimension $n \geq 1$.

(ii) The ample sheaf $\mathscr{L}$ has the property $\mathscr{L}^{\otimes m}=\omega_{B}^{\otimes-1}$ for some $m \geq 1$.

(iii) The group $H^{i}\left(B, \mathscr{L}^{\otimes t}\right)$ vanishes for all integers $i, t \geq 1$.

Then the projective scheme $X$ is Gorenstein, and we have the the Canonical Bundle Formula $K_{X}=-(m+1) A$. In particular, $X$ is a Fano variety of dimension $n+1$, with numerical invariants

$$
\operatorname{deg}(X)=\frac{(m+1)^{n+1}}{m^{n}} \operatorname{deg}(B) \quad \text { and } \quad \operatorname{ind}(X)=m+1 .
$$

Proof. Assumption (iii) guarantees that the cone $X$ is Cohen-Macaulay, according to [Kol13, Proposition 3.13]. In light of Condition (ii) and Proposition 6.3, the dualizing sheaf on the $\mathbb{P}^{1}$-bundle is

$$
\omega_{P}=\mathscr{O}_{P}(-2 E) \otimes f^{*}\left(\mathscr{L}^{\otimes-m-1}\right) .
$$

Furthermore, we have $f^{*}(\mathscr{L})=\mathscr{O}_{P}(A-E)$, which follows from (6) and the exact sequence (5). This gives $K_{P}=-(m+3) E-(m+1) A$. Since $A$ is disjoint from the exceptional locus $E$, we see that $X$ is Gorenstein, having $K_{X}=-(m+1) A$. With Proposition 6.3 we conclude that $X$ is a Fano variety with index $m+1$. The degree is $\left(-K_{X}\right)^{n+1}=(m+1)^{n+1} A^{n+1}$. In light of $(6)$, we have $A^{n+1}=c_{1}^{n}(\mathscr{L})=\frac{1}{m^{n}}\left(-K_{B}\right)^{n}$. This concludes the proof. 


\section{Fano threefolds with unusual torsion}

Let $k$ be an algebraically closed ground field of characteristic $p=2$, and $S$ be a simply-connected Enriques surface endowed with an ADE-curve $E \subset X$ as in Theorem 4.2. This actually exists, as we saw in Section 5. The resulting contraction $f: S \rightarrow Z$ yields a normal Enriques surface, coming with a $G$-torsor $\tilde{Z} \rightarrow Z$, with respect to the Cartier dual $G$ for the unipotent group scheme $P=\operatorname{Pic}_{Z / k}^{\tau}$ of order two. As explained in Section 5, the normalization $Z^{\prime} \rightarrow \tilde{Z}$ gives a del Pezzo surface with $\operatorname{Pic}\left(Z^{\prime}\right)=\mathbb{Z} K_{Z^{\prime}}$ and $h^{1}\left(\mathscr{O}_{Z^{\prime}}\right)=0$, of degree $K_{Z^{\prime}}^{2} \in\{2,4,6,8\}$. It comes with an inseparable double covering $v: Z^{\prime} \rightarrow Z$.

We now consider the $\mathbb{P}^{1}$-bundle $P=\mathbb{P}(\mathscr{E})$ with $\mathscr{E}=\mathscr{O}_{Z^{\prime}} \oplus \omega_{Z^{\prime}}^{\otimes-1}$, and the ensuing contraction

$$
r: P \longrightarrow X=\operatorname{Proj} \bigoplus_{i \geq 0} H^{0}\left(P, \mathscr{O}_{P}(i A)\right)
$$

of the negative section $E=\mathbb{P}\left(\omega_{Z^{\prime}}^{\otimes-1}\right)$, defined via the positive section $A=\mathbb{P}\left(\mathscr{O}_{Z^{\prime}}\right)$, as explained in the previous section. The singular loci can be written as

$$
\operatorname{Sing}(P)=\mathbb{P}_{\text {Sing }\left(Z^{\prime}\right)}^{1} \quad \text { and } \operatorname{Sing}(X)=r\left(\mathbb{P}_{\operatorname{Sing}\left(Z^{\prime}\right)}^{1}\right) .
$$

Proposition 7.1. The scheme $X$ is a normal Fano threefold of degree $\left(-K_{X}\right)^{3}=8 \cdot K_{Z^{\prime}}^{2}$, index $\operatorname{ind}(X)=2$, and $h^{i}\left(\mathscr{O}_{X}\right)=0$ for all integers $i \geq 1$. The Picard scheme is $\operatorname{Pic}_{X / k}=\mathbb{Z}$, which is generated by $\mathscr{O}_{X}(A)$, such that $\omega_{X}=\mathscr{O}_{X}(-2 A)$.

Proof. The statement about the Picard scheme follows from Proposition 6.3. According to Corollary 4.4, we have $h^{1}\left(\omega_{Z^{\prime}}^{\otimes t}\right)=0$. We now can apply Theorem 6.5 with $B=Z^{\prime}, \mathscr{L}=\omega_{Z^{\prime}}$ and $m=1$ and conclude that $X$ is a Fano threefold of index two and degree $\left(-K_{X}\right)^{3}=8 \cdot K_{Z^{\prime}}^{2}$.

It remains to compute the cohomological invariants. The group $H^{1}\left(X, \mathscr{O}_{X}\right)$ is the Lie algebra for the Picard scheme, hence vanishes. Since $X$ is integral and $\omega_{X}^{\otimes-1}$ is ample, the group $H^{0}\left(X, \omega_{X}\right)$ vanishes. Serre duality gives $h^{3}\left(\mathscr{O}_{X}\right)=0$. To see that $h^{2}\left(\mathscr{O}_{X}\right)$ vanishes, it suffices to check $\chi\left(\mathscr{O}_{X}\right)=1$. The Leray-Serre spectral sequences for the fibration $f: P \rightarrow Z^{\prime}$ and the contraction $r: P \rightarrow X$ give

$$
1=\chi\left(\mathscr{O}_{P}\right)=\chi\left(\mathscr{O}_{X}\right)-\chi\left(R^{1} r_{*}\left(\mathscr{O}_{P}\right)\right)+\chi\left(R^{2} r_{*}\left(\mathscr{O}_{P}\right)\right) .
$$

Recall that $E \subset P$ is a negative section that is contracted. The short exact sequence

$$
0 \rightarrow \mathscr{O}_{E}(-n E) \rightarrow \mathscr{O}_{(n+1) E} \rightarrow \mathscr{O}_{n E} \rightarrow 0
$$

yields

$$
H^{i}\left(\mathscr{O}_{E}(-n E)\right) \longrightarrow H^{i}\left(\mathscr{O}_{(n+1) E}\right) \longrightarrow H^{i}\left(\mathscr{O}_{n E}\right) \longrightarrow H^{i+1}\left(\mathscr{O}_{E}(-n E)\right) .
$$

Using the identification $E=Z^{\prime}$ and $\mathscr{O}_{E}(-E)=\omega_{Z^{\prime}}^{\otimes-1}$, we see that $H^{2}\left(\mathscr{O}_{E}(-n E)\right)$ vanishes for all $n \geq 1$. According to Corollary 4.4, the groups $H^{1}\left(\mathscr{O}_{E}(-n E)\right)$ vanish as well. With the Formal Function Theorem we conclude that $R^{1} r_{*}\left(\mathscr{O}_{P}\right)=R^{2} r_{*}\left(\mathscr{O}_{P}\right)=0$, and hence $\chi\left(\mathscr{O}_{X}\right)=1$.

Using the inclusion $Z^{\prime} \subset X$ coming from $Z^{\prime} \subset P \rightarrow X$ and the inseparable double covering $v: Z^{\prime} \rightarrow Z$, we now form the cocartesian and cartesian square

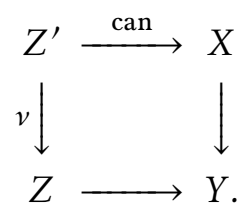

Then $Y=X \amalg_{Z} Z^{\prime}$ is an integral proper threefold, with normalization $X$. A priori, the amalgamated sum exists as an algebraic space ([Art70, Theorem 6.1]). Since the normalization map $v: X \rightarrow Y$ is a universal homeomorphism, the algebraic space $Y$ must be a scheme ([Ols16, Theorem 6.2.2]). It contains the normal Enriques surface $Z$ as a closed subscheme. By construction, the singular locus is given by $\operatorname{Sing}(Y)=Z \cup r\left(\mathbb{P}_{\operatorname{Sing}\left(Z^{\prime}\right)}^{1}\right)$. 
Proposition 7.2. The integral proper threefold $Y$ has the following properties:

(i) The dualizing sheaf $\omega_{Y}$ is invertible and anti-ample, with $\left(-K_{Y}\right)^{3}=K_{Z^{\prime}}^{2}$.

(ii) We have $\operatorname{Num}(Y)=\mathbb{Z}$.

(iii) The Euler characteristic is $\chi\left(\mathscr{O}_{Y}\right)=1$.

(iv) For each closed point $y \in Y$, the local ring $\mathscr{O}_{Y, y}$ satisfies Serre's Condition $\left(S_{2}\right)$. It is actually CohenMacaulay provided that $\mathscr{O}_{Z, y} \subset \mathscr{O}_{Z^{\prime}, y}$ is flat.

Proof. The conductor square (7) yields the short exact sequence

$$
0 \longrightarrow \mathscr{O}_{Y} \longrightarrow \mathscr{O}_{X} \oplus \mathscr{O}_{Z} \longrightarrow \mathscr{O}_{Z^{\prime}} \longrightarrow 0
$$

The normal Enriques surface $Z$ and the normal del Pezzo surface $Z^{\prime}$ both have Euler characteristic $\chi=1$, and the same holds for the normal Fano threefold $X$, by Proposition 7.1. This gives $\chi\left(\mathscr{O}_{Y}\right)=1$.

Since the map $v: X \rightarrow Y$ is surjective, each integral curve on $Y$ is the image of an integral curve on $X$, hence the induced map $v^{*}: \operatorname{Num}(Y) \rightarrow \operatorname{Num}(X)$ is injective, and it follows that the group $\operatorname{Num}(Y)$ is free of rank one.

The singular locus $\operatorname{Sing}(Y)=Z \cup r\left(\mathbb{P}_{\text {Sing }\left(Z^{\prime}\right)}^{1}\right)$ consists of an irreducible surface and a curve. Let $\zeta \in Y$ be the generic point of the conductor locus $Z$, and write $\zeta^{\prime} \in X$ for the corresponding point on $X$. Then $\kappa(\zeta) \subset \mathcal{K}\left(\zeta^{\prime}\right)$ is an inseparable field extension of degree two, and the subring $\mathscr{O}_{Y, \zeta} \subset \mathscr{O}_{X, \zeta}$ comprises all ring elements whose class in the residue field $\kappa\left(\zeta^{\prime}\right)$ lies in the subfield $\kappa(\zeta)$. It follows that the local ring $\mathscr{O}_{Y, \zeta}$ is Gorenstein, compare the discussion in [FS20, Appendix A]. Thus the dualizing sheaf $\omega_{Y}$ is invertible on some open subset containing all codimension-one points. Now let $y \in Y$ be an arbitrary point, and write $x \in X$ for the corresponding point. Since both $\omega_{X}$ and $\mathscr{O}_{X}\left(Z^{\prime}\right)$ are invertible, [FS20, Proposition A.4] applies, and we conclude that the dualizing sheaf $\omega_{Y}$ is invertible.

According to Theorem 6.5, the dualizing sheaf on the Fano threefold $X$ is given by $\omega_{X}=\mathscr{O}_{X}\left(-2 Z^{\prime}\right)$. The relative dualizing sheaf for the finite birational morphism $v: X \rightarrow Y$ is defined by the equality $v_{*}\left(\omega_{X / Y}\right)=\underline{\operatorname{Hom}}\left(v_{*}\left(\mathscr{O}_{X}\right), \mathscr{O}_{Y}\right)=v_{*} \mathscr{O}_{X}\left(-Z^{\prime}\right)$. From $\omega_{X}=v^{*}\left(\omega_{Y}\right) \otimes \omega_{X / Y}$ we conclude $v^{*}\left(\omega_{Y}\right)=\mathscr{O}_{X}\left(-Z^{\prime}\right)$. In particular, $K_{Y}^{3}=\left(-Z^{\prime}\right)^{3}=-K_{Z}^{2}$, holds by Lemma 6.1.

Now fix a closed point $y \in Z$, and consider the three-dimensional local ring $\mathscr{O}_{Y, y}$. It is Cohen-Macaulay by Theorem 6.5 , provided that $y \notin Z^{\prime}$. Now suppose that $y \in Z$. The short exact sequence (8) induces a long exact sequence

$$
H_{y}^{i-1}\left(\mathscr{O}_{Z^{\prime}}\right) \longrightarrow H_{y}^{i}\left(\mathscr{O}_{Y}\right) \longrightarrow H_{y}^{i}\left(\mathscr{O}_{X}\right) \oplus H_{y}^{i}\left(\mathscr{O}_{Z}\right) \longrightarrow H_{y}^{i}\left(\mathscr{O}_{Z^{\prime}}\right)
$$

of local cohomology groups. The two-dimensional schemes $Z$ and $Z^{\prime}$ are Cohen-Macaulay, so their local cohomology groups vanish in degree $i<2$. Likewise, the three-dimensional scheme $X$ is Cohen-Macaulay, so we have vanishing in degree $i<3$. It follows that $H_{y}^{i}\left(\mathscr{O}_{Y}\right)=0$ for $i \leq 1$, hence the local ring $\mathscr{O}_{Y, y}$ satisfies Serre's Condition $\left(S_{2}\right)$.

Finally suppose that $\mathscr{O}_{Z, y} \subset \mathscr{O}_{Z^{\prime}, y}$ is flat. Then the inclusion is a direct summand, in particular the induced map $H_{y}^{2}\left(\mathscr{O}_{Z}\right) \rightarrow H_{y}^{2}\left(\mathscr{O}_{Z}\right)$ is injective. This ensures $H_{y}^{2}\left(\mathscr{O}_{Y}\right)=0$, so the local ring $\mathscr{O}_{Y, y}$ is CohenMacaulay.

We see that the scheme $Y$ qualifies as a Fano variety, except that it is not necessarily Cohen-Macaulay. By definition, a local noetherian ring is called Gorenstein if it is Cohen-Macaulay, and the dualizing module is invertible. Without the former condition, one should use the term quasi-Gorenstein instead. Thus it is natural to call our scheme $Y$ a quasi-Fano variety, or a Fano variety that is not necessarily Cohen-Macaulay. Our construction yields unusual torsion in the Picard scheme: 
Theorem 7.3. The integral Fano threefold $Y$ that is not necessarily Cohen-Macaulay has the following property:

$$
\Upsilon_{Y / k}=\operatorname{Pic}_{Y / k}^{\tau}= \begin{cases}\mathbb{Z} / 2 \mathbb{Z} & \text { if the Enriques surface } S \text { is classical; } \\ \alpha_{2} & \text { if } S \text { is supersingular. }\end{cases}
$$

Moreover, $h^{1}\left(\mathscr{O}_{Y}\right)=h^{2}\left(\mathscr{O}_{Y}\right)=0$ in the former case, and $h^{1}\left(\mathscr{O}_{Y}\right)=h^{2}\left(\mathscr{O}_{Y}\right)=1$ in the latter.

Proof. The conductor square (7) yields a short exact sequence of multiplicative abelian sheaves

$$
1 \rightarrow \mathscr{O}_{Y}^{\times} \rightarrow \mathscr{O}_{X}^{\times} \oplus \mathscr{O}_{Z}^{\times} \rightarrow \mathscr{O}_{Z}^{\times} \rightarrow 1
$$

as explained in [SS02, Proposition 4.1]. This holds not only in the Zariski topology, but also in the finite flat topology. In turn, we get an exact sequence of group schemes

$$
0 \longrightarrow \operatorname{Pic}_{Y / k} \longrightarrow \operatorname{Pic}_{X / k} \oplus \operatorname{Pic}_{Z / k} \longrightarrow \operatorname{Pic}_{Z^{\prime} / k}
$$

The map on the left is indeed injective, because $H^{0}\left(Z, \mathscr{O}_{Z}\right) \rightarrow H^{0}\left(Z^{\prime}, \mathscr{O}_{Z^{\prime}}\right)=k$ is surjective. Since $\rho(Y)=1$, an invertible sheaf $\mathscr{L}$ on $Y$ is numerically trivial if and only if its restriction to $X$, or equivalently to $Z$, is numerically trivial. Since $\rho(X)=1$, the same holds for invertible sheaves on $X$ and their restriction to $Z^{\prime}$. In turn, we get an induced exact sequence

$$
0 \longrightarrow \operatorname{Pic}_{Y / k}^{\tau} \longrightarrow \operatorname{Pic}_{X / k}^{\tau} \oplus \operatorname{Pic}_{Z / k}^{\tau} \longrightarrow \operatorname{Pic}_{Z^{\prime} / k}^{\tau}
$$

The term for the del Pezzo surface $Z^{\prime}$ vanishes, by Theorem 4.2. Also the term for the normal Fano threefold $X$ is zero, according to Proposition 7.1. We thus get an identification $\operatorname{Pic}_{Y / k}^{\tau}=\operatorname{Pic}_{Z / k}^{\tau}$. We saw in Proposition 4.1 that the morphism $S \rightarrow Z$ from the simply-connected Enriques surface $S$ to the normal Enriques surface $Z$ induces an identification $\operatorname{Pic}_{Z / k}^{\tau}=\mathrm{Pic}_{S / k}^{\tau}$ and the assertion on Picard schemes and its maximal unipotent quotient follows.

It remains to check the assertions on $h^{i}\left(\mathscr{O}_{Y}\right)$. This follows from the long exact sequence for (8), together with $h^{i}\left(\mathscr{O}_{X}\right)=h^{i}\left(\mathscr{O}_{Z^{\prime}}\right)=0$ for $i \geq 1$.

Let us now examine a concrete example for the construction of $Y$ : Suppose that our simply-connected Enriques surface $S$ is an exceptional Enriques surface of type $T_{2,3,7}$, and that $f: S \rightarrow Z$ is the contraction of the ADE-curves $C_{1}+\ldots+C_{8}$ and $C_{9}$, as analyzed in Section 5. Recall that $\operatorname{Sing}(Z)=\{a, b\}$ and $\operatorname{Sing}\left(Z^{\prime}\right)=\left\{b^{\prime}\right\}$, where the corresponding local rings are rational double points of type $E_{8}, A_{1}$ and $D_{5}$, respectively. Moreover, the resulting threefold $Y$ has $\operatorname{Sing}(Y)=Z \cup r\left(\mathbb{P}_{b^{\prime}}^{1}\right)$. It turns out that the closed point $a \in Y$, which comes from the $E_{8}$-singularity $\mathscr{O}_{Z, a}$, plays a special role:

Proposition 7.4. In the above situation, our quasi-Fano threefold $Y$ has degree $\left(-K_{Y}\right)^{3}=4$ and $\operatorname{Num}(Y)=\mathbb{Z} K_{Y}$. Moreover, $Y$ is Cohen-Macaulay outside $a \in Y$, whereas the local ring $\mathscr{O}_{Y, a}$ satisfies $\left(S_{2}\right)$ but not $\left(S_{3}\right)$.

Proof. By Corollary 5.4, the double covering $Z^{\prime} \rightarrow Z$ is flat precisely outside $a \in Z$. So Proposition 7.2 tells us that the local rings $\mathscr{O}_{Y, y}$ are Cohen-Macaulay for $y \neq a$. It remains to understand the case $y=a$. Now the cokernel $M$ for the inclusion $\mathscr{O}_{Z, a} \subset \mathscr{O}_{Z^{\prime}, a}$ still is torsion-free of rank one, but fails to be invertible. Since the local ring $\mathscr{O}_{Z, a}$ is a factorial, the bidual $M^{\vee \vee}$ is invertible, and the cokernel $F=M^{\vee \vee} / M$ is finite and non-zero. Using the long exact sequence for the short exact sequence $0 \rightarrow M \rightarrow M^{\vee \vee} \rightarrow F \rightarrow 0$, one easily infers $H_{a}^{1}(M) \neq 0$. With the exact sequence (9) we infer that the map $H_{a}^{2}\left(\mathscr{O}_{Z}\right) \rightarrow H_{a}^{2}\left(\mathscr{O}_{Z^{\prime}}\right)$ is not injective, and hence $H_{a}^{2}\left(\mathscr{O}_{Y}\right) \neq 0$. In turn, the local ring $\mathscr{O}_{Y, a}$ is not Cohen-Macaulay.

Combining Proposition 5.1 and 7.2 we get the degree $\left(-K_{Y}\right)^{3}=K_{Z^{\prime}}^{2}=4$. This is not a multiple of eight, and it follows that $\operatorname{Num}(Y)$ is generated by the canonical class.

By construction, our integral Fano threefold $Y$ is not Cohen-Macaulay in codimension three, and not regular in codimension one. In light of Salomonsson's equations [Sal03], the construction works over any ground field of characteristic $p=2$. It would be interesting to know if there are imperfect fields $F$ over which $Y$ admits a twisted form $Y^{\prime}$ whose local rings are normal, $\mathbb{Q}$-factorial klt singularities. Such 
twisted forms could appear as generic fibers in Mori fiber spaces. Recall that there are indeed examples of three-dimensional normal, $\mathbb{Q}$-factorial terminal singularities that are not Cohen-Macaulay [Tot19]. In [Sch07], related problems where considered for non-normal del Pezzo surface. Del Pezzo surfaces over $F$ with $\operatorname{pdeg}(F)=1$ were studied in [FS20]. Here the $p$-degree is defined as $\operatorname{pdeg}(F)=\operatorname{dim}_{F}\left(\Omega_{F / F p}^{1}\right)$.

\section{References}

[AH19] Jeffrey D. Achter and Everett W. Howe, Hasse-Witt and Cartier-Manin matrices: a warning and a request, Arithmetic geometry: computation and applications, Contemp. Math. 722, Amer. Math. Soc., Providence, RI, 2019, pp. 1-18.

[Art66] Michael Artin, On isolated rational singularities of surfaces, Amer. J. Math. 88 (1966), 129-136.

[Art70] M. Artin, Algebraization of formal moduli. II. Existence of modifications, Ann. of Math. (2) 91 (1970), $88-135$.

[Art77] M. Artin, Coverings of the rational double points in characteristic $p$, Complex analysis and algebraic geometry, 1977, pp. 11-22.

[BE95] Dave Bayer and David Eisenbud, Ribbons and their canonical embeddings, Trans. Amer. Math. Soc. 347 (1995), no. 3, 719-756.

[Ber19] Fabio Bernasconi, Non-normal purely log terminal centres in characteristic $p \geqslant 3$, Eur. J. Math. 5 (2019), no. 4, 1242-1251.

[BLR90] Siegfried Bosch, Werner Lütkebohmert, and Michel Raynaud, Néron models, Ergebnisse der Mathematik und ihrer Grenzgebiete (3), vol. 21, Springer-Verlag, Berlin, 1990.

[BM76] E. Bombieri and D. Mumford, Enriques' classification of surfaces in char. p. III, Invent. Math. 35 (1976), 197-232.

[Bou83] Nicolas Bourbaki, Éléments de mathématique, Masson, Paris, 1983, Algèbre commutative. Chapitre 8. Dimension. Chapitre 9. Anneaux locaux noethériens complets.

[Bri09] Michel Brion, Anti-affine algebraic groups, J. Algebra 321 (2009), no. 3, 934-952.

[Bril7] Michel Brion, Some structure theorems for algebraic groups, Algebraic groups: structure and actions, Proc. Sympos. Pure Math., vol. 94, Amer. Math. Soc., Providence, RI, 2017, pp. 53-126.

[BT20] Fabio Bernasconi and Hiromu Tanaka, On Del Pezzo fibrations in positive characteristic, Journal of the Institute of Mathematics of Jussieu (2020), 1-43.

[CD89] François R. Cossec and Igor V. Dolgachev, Enriques surfaces. I, Progress in Mathematics 76, Birkhäuser Boston, Inc., Boston, MA, 1989.

[CT19] Paolo Cascini and Hiromu Tanaka, Purely log terminal threefolds with non-normal centres in characteristic two, Amer. J. Math. 141 (2019), no. 4, 941-979.

[DG70] Michel Demazure and Pierre Gabriel, Groupes algébriques. Tome I: Géométrie algébrique, généralités, groupes commutatifs, Masson, Paris, 1970.

[DI87] Pierre Deligne and Luc Illusie, Relèvements modulo $p^{2}$ et décomposition du complexe de de Rham, Invent. Math. 89 (1987), no. 2, 247-270.

[Dol12] Igor V. Dolgachev, Classical algebraic geometry, Cambridge University Press, Cambridge, 2012.

[EGA2] A. Grothendieck, Éléments de géométrie algébrique. II. Étude globale élémentaire de quelques classes de morphismes, Inst. Hautes Études Sci. Publ. Math. 8 (1961), 5-222. 
[EGA4-II] A. Grothendieck, Éléments de géométrie algébrique. IV. Étude locale des schémas et des morphismes de schémas. II, Inst. Hautes Études Sci. Publ. Math. 24 (1965), 5-231.

[EGA4-III] A. Grothendieck, Éléments de géométrie algébrique. IV. Étude locale des schémas et des morphismes de schémas. III, Inst. Hautes Études Sci. Publ. Math. 28 (1966), 5-255.

[Ein00] Lawrence Ein, Linear systems with removable base loci, Comm. Algebra 28 (2000), no. 12, special issue in honor of Robin Hartshorne, pp. 5931-5934.

[ESB04] Torsten Ekedahl and Nicholas Shepherd-Barron, On exceptional Enriques surfaces, preprint arXiv:math.AG/0405510 (2004).

[Fan31] G. Fano, Sulle varietà algebriche a tre dimensioni aventi tutti i generi nulli., Atti Congresso Bologna 4 (1931), 115-121.

[FGA4] Alexander Grothendieck, Technique de descente et théorèmes d'existence en géométrie algébrique. VI. Les schémas de Picard: propriétés générales, Séminaire Bourbaki, Vol. 7, Soc. Math. France, Paris, 1962, pp. Exp. No. 236, 221-243.

[FS20] Andrea Fanelli and Stefan Schröer, Del Pezzo surfaces and Mori fiber spaces in positive characteristic, Trans. Amer. Math. Soc. 373 (2020), no. 3, 1775-1843.

[Fuj83] Takao Fujita, Semipositive line bundles, J. Fac. Sci. Univ. Tokyo Sect. IA Math. 30 (1983), no. 2, 353-378.

[Har77] Robin Hartshorne, Algebraic geometry, Springer-Verlag, New York-Heidelberg, 1977, Graduate Texts in Mathematics, No. 52.

[Har94] Robin Hartshorne, Generalized divisors on Gorenstein schemes, Proceedings of Conference on Algebraic Geometry and Ring Theory in honor of Michael Artin, Part III (Antwerp, 1992), vol. 8, 1994, pp. 287-339.

[HW36] Helmut Hasse and Ernst Witt, Zyklische unverzweigte Erweiterungskörper vom Primzahlgrade p über einem algebraischen Funktionenkörper der Charakteristik p, Monatsh. Math. Phys. 43 (1936), no. 1, 477-492.

[Jou83] Jean-Pierre Jouanolou, Théorèmes de Bertini et applications, Progress in Mathematics, vol. 42, Birkhäuser Boston, Inc., Boston, MA, 1983.

[Kle66] Steven L. Kleiman, Toward a numerical theory of ampleness, Ann. of Math. (2) 84 (1966), 293-344.

[Kol13] János Kollár, Singularities of the minimal model program, Cambridge Tracts in Mathematics, vol. 200, Cambridge University Press, Cambridge, 2013, With a collaboration of Sándor Kovács.

[Mad16] Zachary Maddock, Regular del Pezzo surfaces with irregularity, J. Algebraic Geom. 25 (2016), no. 3, 401-429.

[Mum61] David Mumford, The topology of normal singularities of an algebraic surface and a criterion for simplicity, Inst. Hautes Études Sci. Publ. Math. 9 (1961), 5-22.

[Mum66] David Mumford, Lectures on curves on an algebraic surface, with a section by G. M. Bergman. Annals of Mathematics Studies, No. 59, Princeton University Press, Princeton, N.J., 1966.

[Nik80] V. V. Nikulin, Integral symmetric bilinear forms and some of their applications., Math. USSR, Izv. 14 (1980), 103-167 (English).

[Ols16] Martin Olsson, Algebraic spaces and stacks, American Mathematical Society Colloquium Publications, vol. 62, American Mathematical Society, Providence, RI, 2016. 
[Oor66] Frans Oort, Commutative group schemes, Lecture Notes in Mathematics, vol. 15, Springer-Verlag, Berlin-New York, 1966.

[PW17] Zsolt Patakfalvi and Joe Waldron, Singularities of General Fibers and the LMMP, Preprint arXiv:1708.04268 (2017).

[Ray70] M. Raynaud, Spécialisation du foncteur de Picard, Inst. Hautes Études Sci. Publ. Math. 38 (1970), $27-76$.

[Rei94] Miles Reid, Nonnormal del Pezzo surfaces, Publ. Res. Inst. Math. Sci. 30 (1994), no. 5, 695-727.

[Rus70] Peter Russell, Forms of the affine line and its additive group, Pacific J. Math. 32 (1970), 527-539.

[Sal03] Pelle Salomonsson, Equations for some very special Enriques surfaces in characteristic two, preprint arXiv:math/0309210 (2003).

[SB97] N. I. Shepherd-Barron, Fano threefolds in positive characteristic, Compositio Math. 105 (1997), no. 3, 237-265.

[Sch01] Stefan Schröer, Normal del Pezzo surfaces containing a nonrational singularity, Manuscripta Math. 104 (2001), no. 2, 257-274.

[Sch07] Stefan Schröer, Weak del Pezzo surfaces with irregularity, Tohoku Math. J. (2) 59 (2007), no. 2, 293-322.

[Sch09] Stefan Schröer, The Hilbert scheme of points for supersingular abelian surfaces, Ark. Mat. 47 (2009), no. 1, 143-181.

[Sch10] Stefan Schröer, On fibrations whose geometric fibers are nonreduced, Nagoya Math. J. 200 (2010), $35-57$.

[Sch17] Stefan Schröer, Enriques surfaces with normal K3-like coverings, preprint arXiv:1703.03081 (2017).

[Sch19] Stefan Schröer, A higher-dimensional generalization of Mumford's rational pullback for Weil divisors, J. Singul. 19 (2019), 53-60.

[Ser58] Jean-Pierre Serre, Sur la topologie des variétés algébriques en caractéristique $p$, Symposium internacional de topología algebraica, Universidad Nacional Autónoma de México and UNESCO, Mexico City, 1958, pp. 24-53.

[SGA1] Alexandre Grothendieck (ed), Revêtements étales et groupe fondamental, Lecture Notes in Mathematics 224, Springer-Verlag, Berlin-New York, 1971, Séminaire de Géométrie Algébrique du Bois Marie 1960-1961 (SGA 1).

[SGA3-I] M. Demazure and A. Grothendieck (eds), Schémas en groupes. I: Propriétés générales des schémas en groupes, Séminaire de Géométrie Algébrique du Bois Marie 1962/64 (SGA 3). Lecture Notes in Mathematics 151, Springer-Verlag, Berlin-New York, 1970.

[SGA3-II] M. Demazure and A. Grothendieck (eds), Schémas en groupes. II: Groupes de type multiplicatif, et structure des schémas en groupes généraux, Séminaire de Géométrie Algébrique du Bois Marie 1962/64 (SGA 3). Lecture Notes in Mathematics 152, Springer-Verlag, Berlin-New York, 1970.

[SGA4-II] M. Artin, A. Grothendieck and J.-L. Verdier (eds), Théorie des topos et cohomologie étale des schémas. Tome 2, Lecture Notes in Mathematics 270, Springer-Verlag, Berlin-New York, 1972, Séminaire de Géométrie Algébrique du Bois-Marie 1963-1964 (SGA 4).

[SGA6] P. Berthelot, A. Grothendieck and L. Illusie (eds), Théorie des intersections et théorème de RiemannRoch, Lecture Notes in Mathematics 225, Springer-Verlag, Berlin-New York, 1971, Séminaire de Géométrie Algébrique du Bois-Marie 1966-1967 (SGA 6). 
[SS02] Stefan Schröer and Bernd Siebert, Irreducible degenerations of primary Kodaira surfaces, Complex geometry (Göttingen, 2000), Springer, Berlin, 2002, pp. 193-222.

[SV04] Stefan Schröer and Gabriele Vezzosi, Existence of vector bundles and global resolutions for singular surfaces, Compos. Math. 140 (2004), no. 3, 717-728.

[Tan16] Hiromu Tanaka, Pathologies on Mori fibre spaces in positive characteristic, preprint arXiv:1609.00574 (2016).

[Tan18] Hiromu Tanaka, Behavior of canonical divisors under purely inseparable base changes, J. Reine Angew. Math. 744 (2018), 237-264.

[Tot19] Burt Totaro, The failure of Kodaira vanishing for Fano varieties, and terminal singularities that are not Cohen-Macaulay, J. Algebraic Geom. 28 (2019), no. 4, 751-771.

[WITO69] Kei-ichi Watanabe, Takeshi Ishikawa, Sadao Tachibana, and Kayo Otsuka, On tensor products of Gorenstein rings, J. Math. Kyoto Univ. 9 (1969), 413-423.

[Yas19] Takehiko Yasuda, Discrepancies of p-cyclic quotient varieties, J. Math. Sci. Univ. Tokyo 26 (2019), no. 1, $1-14$. 